\title{
Amplified engagement of prefrontal cortex during control of voluntary action in Tourette syndrome
}

\author{
(DCharlotte L. Rae, ,,2 Jim Parkinson, ',2 Sophie Betka, 3,4,5,6 \\ Cassandra D. Gouldvan Praag, ${ }^{3,7}$ Samira Bouyagoub, ${ }^{3}$ Liliana Polyanska, 3,8,9 \\ (D)Dennis E. O. Larsson, ${ }^{1,2,3}$ DNeil A. Harrison, ${ }^{3,10}$ Sarah N. Garfinkel ${ }^{2,3, I I}$ and \\ Hugo D. Critchley $2,3, I^{\prime}$
}

Tourette syndrome is characterized by 'unvoluntary' tics, which are compulsive, yet often temporarily suppressible. The inferior frontal gyrus is implicated in motor control, including inhibition of pre-potent actions through influences on downstream subcortical and motor regions. Although tic suppression in Tourette syndrome also engages the inferior frontal gyrus, it is unclear whether such prefrontal control of action is also dysfunctional: Tic suppression studies do not permit comparison with control groups, and neuroimaging studies of motor inhibition can be confounded by the concurrent expression or suppression of tics. Here, patients with Tourette syndrome were directly compared to control participants when performing an intentional inhibition task during functional MRI. Tic expression was recorded throughout for removal from statistical models. Participants were instructed to make a button press in response to Go cues, withhold responses to NoGo cues, and decide whether to press or withhold to 'Choose' cues. Overall performance was similar between groups, for both intentional inhibition rates (\% Choose-Go) and reactive NoGo inhibition commission errors. A subliminal face prime elicited no additional effects on intentional or reactive inhibition. Across participants, the task activated prefrontal and motor cortices and subcortical nuclei, including pre-supplementary motor area, inferior frontal gyrus, insula, caudate nucleus, thalamus and primary motor cortex. In Tourette syndrome, activity was elevated in the inferior frontal gyrus, insula and basal ganglia, most notably within the right inferior frontal gyrus during voluntary action and inhibition (Choose-Go and Choose-NoGo), and reactive inhibition (NoGo-correct). Anatomically, the locus of this inferior frontal gyrus hyperactivation during control of voluntary action matched that previously reported for tic suppression. In Tourette syndrome, activity within the caudate nucleus was also enhanced during both intentional (Choose-NoGo) and reactive (NoGo-correct) inhibition. Strikingly, despite the absence of overt motor behaviour, primary motor cortex activity increased in patients with Tourette syndrome but decreased in controls during both reactive and intentional inhibition. Additionally, severity of premonitory sensations scaled with functional connectivity of the pre-supplementary motor area to the caudate nucleus, globus pallidus and thalamus when choosing to respond (Choose-Go). Together, these results suggest that patients with Tourette syndrome use equivalent prefrontal mechanisms to suppress tics and withhold non-tic actions, but require greater inferior frontal gyrus engagement than controls to overcome motor drive from hyperactive downstream regions, notably primary motor cortex. Moreover, premonitory sensations may cue midline motor regions to generate tics through interactions with the basal ganglia.

1 School of Psychology, University of Sussex, Sussex BN1 9QH, UK

2 Sackler Centre for Consciousness Science, University of Sussex, Sussex, UK

3 Department of Neuroscience, Brighton \& Sussex Medical School, Sussex BN1 9RY, UK

4 Laboratory of Cognitive Neuroscience, Center for Neuroprosthetics \& Brain Mind Institute, Ecole Polytechnique Fédérale de Lausanne, Geneva CH-1202, Switzerland

5 Neurosurgery Division, Geneva University Hospitals, Geneva 1205, Switzerland

Received February 9, 2020. Revised October 9, 2020. Accepted October 13, 2020. Advance Access publication November 27, 2020 (C) The Author(s) (2020). Published by Oxford University Press on behalf of the Guarantors of Brain.

This is an Open Access article distributed under the terms of the Creative Commons Attribution Non-Commercial License (http://creativecommons.org/licenses/by-nc/4.0/), which permits non-commercial re-use, distribution, and reproduction in any medium, provided the original work is properly cited. For commercial re-use, please contact journals.permissions@oup.com 
6 Department of Clinical Neurosciences, Geneva University Hospitals, Geneva 1205, Switzerland

7 Department of Psychiatry, University of Oxford, Oxford OX3 7JX, UK

8 Center for Lifespan Psychology, Max Planck Institute for Human Development, Berlin 14195, Germany

9 Department of Education and Psychology, Freie Universität Berlin, Berlin 14195, Germany

10 CUBRIC, University of Cardiff, Cardiff CF24 4HQ, UK

11 Sussex Partnership NHS Foundation Trust, Sussex BN3 7HZ, UK

Correspondence to: Dr. Charlotte L Rae, School of Psychology, Pevensey Building, University of

Sussex, Falmer BN1 9QH, UK

E-mail: c.rae@sussex.ac.uk

Keywords: basal ganglia; movement disorders; neuropsychiatry; tic disorder; Tourette's syndrome

Abbreviations: ADHD = attention deficit hyperactivity disorder; ASRS = Adult ADHD Self-Report Scale; FDR = false-discovery rate; $\mathrm{fMRI}=$ functional $\mathrm{MRI}$;FG = inferior frontal gyrus; $\mathrm{M} 1=$ primary motor cortex; $\mathrm{OCD}=$ obsessive compulsive disorder; PPI = psychophysiological interaction; preSMA = pre-supplementary motor area; PUTS = Premonitory Urge for Tics Scale; YBOCS $=$ Yale Brown Obsessive Compulsive Scale; YGTSS = Yale Global Tic Severity Scale

\section{Graphical Abstract}

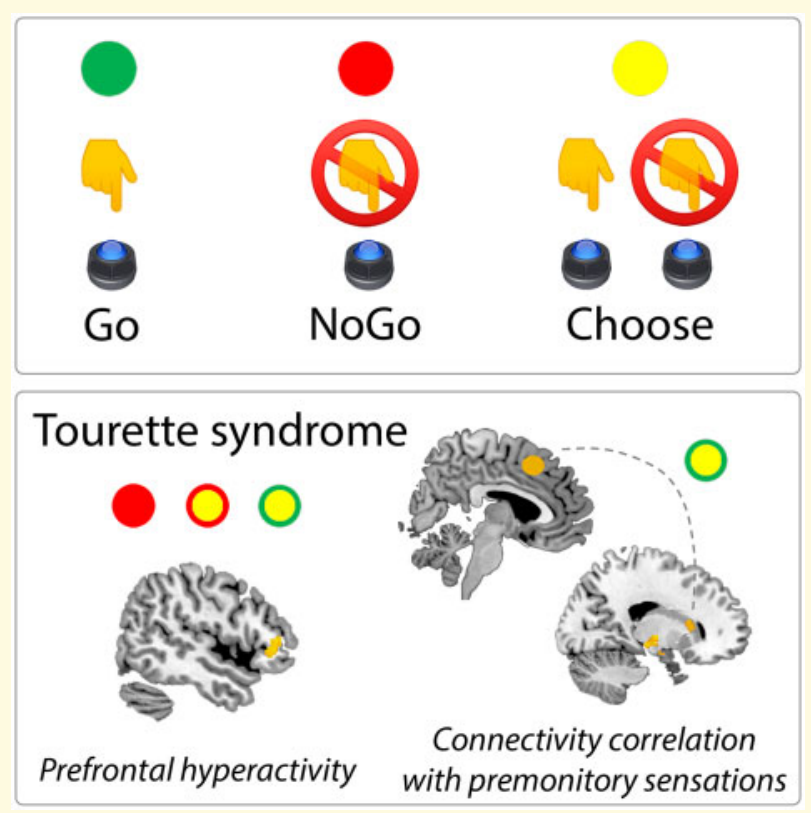

\section{Introduction}

Tourette syndrome is a neurodevelopmental hyperkinetic movement disorder, characterized by motor and phonic tics. A curious feature of these tics is that they are often reported to be semi-voluntary reactions to premonitory urges to move (Kwak et al., 2003; Cavanna and Nani, 2013; Cavanna et al., 2017). Furthermore, patients with Tourette syndrome can often temporarily withhold or suppress them, for example, in social contexts (Matsuda et al., 2016). This suggests at least partial volitional control and is distinctive from other hyperkinetic disorders, such as myoclonus (Ganos et al., 2018b). Behavioural therapies, such as exposure and response prevention, capitalize on this capacity for voluntary tic suppression to habituate to and diminish premonitory urges (Frundt et al., 2017; Ganos et al., 2018b).
Dysfunctional interactions within cortico-striato-thalamo-cortical circuits are believed to underlie tic generation (Ganos, 2016) and prefrontal cortex is implicated in their control. For example, functional imaging studies report enhanced activity within lateral prefrontal cortex, particularly the (anterior) inferior frontal gyrus (IFG) during tic suppression (Peterson et al., 1998; Ganos et al., 2014a). This indicates that the active control of tics in Tourette syndrome likely engages the same prefrontal mechanisms as are implicated in stopping or withholding (non-tic) actions (Ganos et al., 2014b; Zapparoli et al., 2015; Rae et al., 2019). Interestingly, patients with Tourette syndrome show greater IFG activity (Zapparoli et al., 2015), and reduced functional connectivity of primary motor cortex, on (non-tic) response inhibition tasks (Thomalla et al., 2014). Together, this suggests that the IFG supports both reactive motor inhibition and 
volitional tic suppression, in accordance with theoretical notions that volitional tic inhibition may overlap with other forms of motor inhibitory processes, with a 'neural signature of both internally decided and externally triggered inhibition', centred on the IFG (Ganos et al., 2018b). However, in Tourette syndrome, greater prefrontal engagement may be necessary to overcome hyper-activity in motor output regions including primary motor cortex (Ganos, 2016; Rae et al., 2019).

The pre-supplementary motor area (preSMA) is also central to action inhibition, interacting with signals from IFG to modulate basal ganglia activity (Rae et al., 2015; Aron et al., 2016). The pre-supplementary motor area is a cardinal substrate for voluntary action decisions, across 'what-when-whether' categories (Brass and Haggard, 2008; Zapparoli et al., 2017a). However, functional MRI (fMRI) studies of tic expression show supplementary motor area (SMA) rather than preSMA activity prior to tic release (Bohlhalter et al., 2006; Neuner et al., 2014; Zapparoli et al., 2015). This raises questions of whether preSMA activity is altered in Tourette syndrome during the control of voluntary action, and how interactions with the basal ganglia affect tic expression. Functional MRI studies characterizing neural processes in Tourette syndrome are rarely free of interpretive confounds. Overt tic suppression studies cannot meaningfully compare patients' brain activity during suppression periods to controls who do not tic (van der Salm et al., 2018). In addition, accidental expression of tics during instructed suppression periods may confound the interpretation of tic suppression versus 'free ticcing' studies, whereas the results in task-based studies may be affected by covert tic suppression. Instead, 'intentional inhibition' tasks enable direct comparison of Tourette syndrome and control participants, revealing for example, heightened dorsal anterior cingulate and striatal activity in Tourette syndrome when participants are instructed to inhibit eye blinks (and suppress ocular tics) (Mazzone et al., 2010; van der Salm et al., 2018).

Here, we used an intentional inhibition task during fMRI to compare participants with Tourette syndrome and controls. However, rather than instructing participants not to suppress tics, we used video-monitoring time-locked to fMRI acquisition to identify tics. We then used this to construct participant-specific tic regressors to isolate activity related to tic expression from task performance. This approach enabled examination of task effects uncontaminated by tic expression or suppression (Neuner et al., 2007; Thomalla et al., 2014; Rae et al., 2018).

In addition, we used a modified Go/NoGo task, to incorporate 'Choose' trials, when participants chose whether to act or to withhold a button press (Rae et al., 2020). This allowed simultaneous investigation of voluntary action (on Choose trials when participants elected to act), intentional inhibition (on Choose trials when participants elected to withhold) and reactive inhibition (on
NoGo trials). The task also balanced the number of trials across participants, in contrast to tic or blink suppression paradigms, where statistical power may be compromised by subject-level variance in frequency of tics or tic suppression, during scanning.

We predicted that participants with Tourette syndrome show similar patterns of anatomical engagement to controls, in line with the hypothesis that tic suppression uses the same core circuitry for stopping and withholding of (non-tic) movements (Rae et al., 2019). Moreover, we predicted that prefrontal and motor control sites would be hyperactive in Tourette syndrome, against the backdrop of basal ganglia dysfunction and elevated primary motor cortex reactivity (Jackson et al., 2015; Zapparoli et al., 2017b). Finally, we predicted that the strength of interaction between prefrontal and cortical motor planning regions (notably IFG and pre-SMA) with basal ganglia nuclei would determine the severity of Tourette syndrome symptoms (Thomalla et al., 2014; Zapparoli et al., 2017b; Rae et al., 2018).

We previously identified hyperactivity of insular cortex and stronger functional connectivity between insula and motor areas when patients with Tourette syndrome view faces, suggesting that the insula can trigger tic expression during social stimulation (Rae et al., 2018). The insula is also active during 'whether' decisions to act or to withhold (Brass and Haggard, 2010), suggesting that this region may also cue such motor decisions. We therefore included within our task design a subliminal priming element (Parkinson et al., 2017). We hypothesized that unconscious facial primes might differentially cue 'Choose' decisions to act or to withhold responses through effects on insular activation.

\section{Materials and methods}

\section{Participants}

Twenty-three participants with Tourette syndrome (13 male [age, 18-51 years], mean 34 years) and 21 controls with no history of major neurological or psychiatric disorder (11 male [age, 19-55 years], mean 35 years) participated. Clinical diagnosis of Tourette syndrome was made by a UK neurologist or psychiatrist specialized in the assessment of Tourette syndrome. Patients were recruited from the Sussex Partnership NHS Foundation Trust Neurodevelopmental Service (psychiatrist H.D.C.), and via Tourettes Action UK (specifying details of their clinical assessment prior to inclusion). Obsessive compulsive disorder (OCD) and attention deficit hyperactivity disorder (ADHD) diagnoses were also recorded.

Tic severity was assessed using the Yale Global Tic Severity Scale (YGTSS, symptom severity: maximum 50; impairment: maximum 50; global total: 100) (Leckman et al., 1989). fMRI analyses used the symptom severity score. Premonitory sensations were assessed using the 
Premonitory Urge for Tics Scale (PUTS, Woods et al., 2005); OCD severity using the Yale Brown Obsessive Compulsive Scale (YBOCS, Goodman et al., 1989) and ADHD severity using the Adult ADHD Self-Report Scale (ASRS, Kessler et al., 2005).

Two patients were taking dopaminergic medications, six serotonergic medications and one was taking both dopaminergic and serotonergic medications. One patient on sertraline was also taking a benzodiazepine. The remaining 14 were unmedicated (one of whom took melatonin as a natural sleep aid remedy).

Table 1 gives demographic details and clinical features (Supplementary Table 1: individual patient data). Participants gave written informed consent. The study was approved by the South East Coast: Brighton National Research Ethics Committee (15-LO-0109).

\section{Intentional inhibition task}

Participants performed a modified Go/NoGo task in which movement cues (green, red and yellow circles) were presented on a grey background for $800 \mathrm{~ms}$ (Fig. 1). Green Go cues indicated a button press to be made with the right index finger, red NoGo cues indicated the participant should withhold their button press and yellow 'Choose' cues indicated participants should choose whether to press the button or withhold (Rae et al., 2020). There were 864 trials: 432 Go (50\%), 144 NoGo $(17 \%)$ and 288 Choose $(33 \%)$, presented in a pseudorandomized order. The higher frequency of Go trials was designed to invoke a pre-potent tendency to go, as in traditional Go/NoGo tasks and ensure that withholding on NoGo trials was sufficiently challenging to invoke inhibitory control (Rae et al., 2020). Participants were instructed to respond quickly on Go trials, withhold button presses on NoGo trials and choose quickly, making a fresh decision each time, on Choose trials.

To test the secondary hypothesis regarding social context on motor decisions, each trial also involved subliminal presentation of a face prime prior to each movement cue. These face primes (from the NIMSTIM database; (Tottenham et al., 2009)) portrayed (i) neutral (33\%) or (ii) angry $(33 \%)$ expressions or were (iii) scrambled

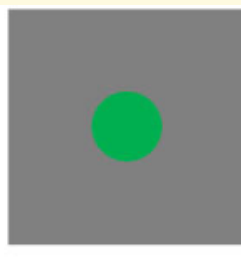

Go

(50\%)

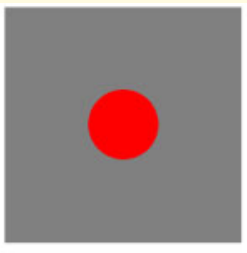

NoGo

(17\%)

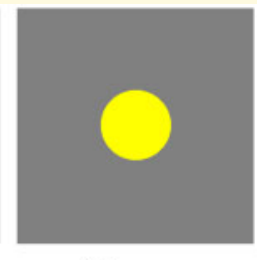

Choose

(33\%)
Figure I Intentional inhibition task cues. Following an intertrial interval, on Go trials (50\%) green cues instructed participants to make a button press, on NoGo trials (17\%) red cues instructed participants to withhold, and on Choose trials (33\%) yellow cues indicated participants should choose whether to press or withhold. Stimuli enlarged for illustrative purposes.

Table I Demographic details of participants, clinical features of patients and behavioural performance on the intentional inhibition task

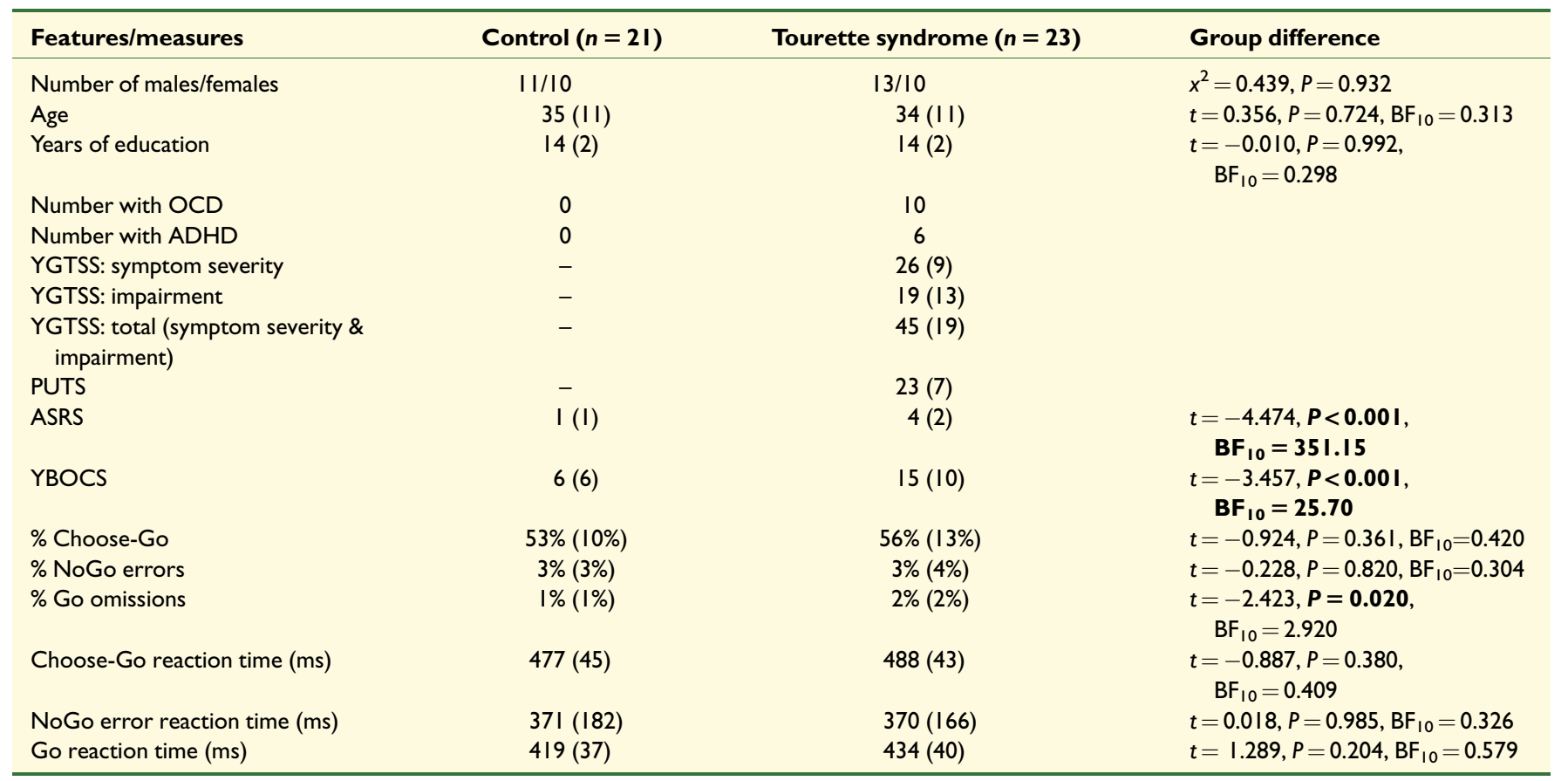

Data are presented as means (SD). Group difference $P$-values refer to two-tailed $t$-tests or Chi-squared for number of males/females.

OCD, obsessive compulsive disorder; ADHD, attention deficit hyperactivity disorder; YGTSS, Yale Global Tic Severity Scale; PUTS, Premonitory Urge for Tics Scale; YBOCS, YaleBrown Obsessive Compulsive Scale; ASRS, Adult ADHD Self-Report Scale. 
$(33 \%)$ for a 'non-social' control. Three male and three female identities were used over 144 trials (72 Go, 24 NoGo and 48 Choose). Hair and peripheral features were removed by applying a greyscale circle, leaving only the facial expression. The face primes, presented for $16 \mathrm{~ms}$, were preceded by a scrambled face 'forward mask' (32 ms), followed by a scrambled face 'backward mask' (48 ms), to render the face prime consciously imperceptible (Parkinson et al., 2017). Participants were not informed of the face primes before or during the experiment.

A white fixation cross was displayed during inter-trial intervals, jittered in duration and optimized using OptSeq (http://surfer.nmr.mgh.harvard.edu/optseq; accessed 30 November 2020) for event-related design efficiency $(35 \%$ $1000 \mathrm{~ms}, 30 \% 1130 \mathrm{~ms}, 20 \% 1250 \mathrm{~ms}, 10 \% 1380 \mathrm{~ms}$ and $5 \% 1500 \mathrm{~ms})$. The task was divided into three blocks of 288 trials, $10 \mathrm{~min} 42 \mathrm{~s}$ in duration, with breaks in between to reduce fatigue and discomfort.

\section{Intentional inhibition task statistical analysis}

Indices of motor behaviour (proportion of Choose trials on which participants decided to act i.e. \% Choose-Go, NoGo commission errors, Go omissions and reaction times) were compared between Tourette syndrome patients and controls using independent-sample $t$-tests and Bayesian equivalents (applying default priors) in JASP (https://jasp-stats.org; accessed 30 November 2020). We examined the effects of face priming on intentional inhibition ( $\%$ Choose-Go) using a $3 \times 2$ repeated measures ANOVA (within group factor, face prime: neutral, angry, scrambled; between groups factor: TS, control), and an equivalent Bayesian ANOVA (comparing to a null model). To examine whether task performance changed over the duration of the experiment, and whether this differed between patients and controls, we compared the six motor behaviour indices (\% Choose-Go, NoGo commission errors, Go omissions and reaction times) in the first block of trials $(1-288)$ to the final block (trials 577-864) using six $2 \times 2$ repeated measures ANOVAs (within group factor: block 1, block 3; between groups factor: TS, control) and equivalent Bayesian ANOVAs (comparing to a null model).

\section{Face prime subliminality assessment}

To verify that face primes were consciously imperceptible, after the intentional inhibition task two brief behavioural checks were employed (Supplementary Methods).

\section{MRI acquisition}

The fMRI data were acquired on a Siemens Avanto $1.5 \mathrm{~T}$ (32 channel head coil, T2*-weighted images, repetition time $=2520 \mathrm{~ms}$, echo time $=43 \mathrm{~ms}, 34$ ascending $3 \mathrm{~mm}$ slices, $0.6 \mathrm{~mm}$ slice gap, in-plane resolution $3 \times 3 \mathrm{~mm}$ ). In total, $255 \mathrm{fMRI}$ volumes were acquired per 10-min block (total, 765 volumes). The first five volumes per block were discarded for steady-state magnetization. A T1weighted image was acquired for fMRI preprocessing (repetition time $=2730 \mathrm{~ms}$, echo time $=3.57 \mathrm{~ms}$, $1 \times 1 \times 1 \mathrm{~mm}$ resolution). Participants' heads were tightly cushioned within the head coil to reduce head movements.

\section{Tic monitoring}

We did not instruct participants to suppress tics. This was essential to acquire intentional inhibition task fMRI data uncontaminated by simultaneous tic suppression in Tourette syndrome participants. Furthermore, not instructing participants to suppress tics reduces distress and fatigue over the imaging session. To remove BOLD signal relating to generation and expression of tics during the task, we video-recorded tics, time-locked to fMRI data and included tic expression as a regressor in general linear modelling (Rae et al., 2018). Videos were recorded concurrently with acquisition of neuroimaging data in order to identify the timings of tics, which were used to exclude the effects of tic generation and expression from neuroimaging analyses, but were not used to rate tic severity.

We acquired video using an in-bore MRI compatible camera (MRC Systems, www.mrc-systems.de; accessed 30 November 2020), mounted on the head coil to view participants' faces, and an out-of-bore camera to view limbs and body $(360 \times 240$ resolution, 30 frames/s $)$. Camera feeds and fMRI volume markers were simultaneously relayed to Spike2 physiological recording software (version 7.17, CED). During fMRI acquisition, the researcher (C.L.R.) watched the live video feeds and noted fMRI volumes at which she observed tics within a written record, in case the video recordings were interrupted, lost or failed in another way. Storage of the video recording failed for three participants; in these cases, the written records alone identified tic onsets and durations in relation to the fMRI time series.

For the majority of participants with complete video recordings $(n=20)$, tics were identified in post-hoc video assessment, using the written record as a supplementary guide. Initial tic ratings were conducted by two authors (L.P.: 8 videos, D.E.O.L.:12), before a second rater, familiar with each patient's tic repertoire (C.L.R.), conducted a second rating, confirming or rejecting the status of each event as a tic, and identifying any not previously flagged by L.P. or D.E.O.L. An in-house Spike2 script extracted tic onsets and durations, time-locked to fMRI data. Phonic tics were often visible from facial movement, but we did not record sound.

During the $30 \mathrm{~min}$ of fMRI, an average of 161 tics occurred (range, 0-551, standard deviation: 147). The bodily locations at which tics were expressed were on 
average $40 \%$ facial, $8 \%$ head, $8 \%$ both face and head, $33 \%$ body or limbs and $11 \%$ combinations of face, head, body and limbs.

\section{fMRI preprocessing}

The fMRI data were preprocessed and analysed using SPM12 (v7219, www.fil.ion.ucl.ac.uk/spm; accessed 30 November 2020). Pre-processing used default options, including realignment to the mean image, slice-time correction to the middle slice, co-registration with T1 structural and MNI normalization and 8-mm smoothing.

\section{Task fMRI univariate statistical analysis}

A general linear model represented task events, with regressors for onset and duration $(500 \mathrm{~ms})$ of (i) Go, (ii) NoGo-correct, (iii) Choose-Go and (iv) Choose-NoGo trials. If participants made Go omissions or NoGo errors, these regressors were added. Because there were no effects of face primes on behaviour (Results section), we collapsed across prime types for all imaging analyses. The general linear model of Tourette syndrome participants contained a further regressor for observed onsets and durations of tics. The fMRI data from the three runs were concatenated (spm_concatenate.m), adding a constant (mean) column for each of the three runs and a 'block transitions' regressor modelled the transition from end of one block to the start of the next. Six realignment parameter regressors modelled head movement.

Single-regressor $T$-contrasts were generated for (i) Go, (ii) NoGo-correct, (iii) Choose-Go and (iv) Choose-Nogo trials, and NoGo errors if made, with implicit baseline of inter-trial interval fixation cross. These were entered to a full factorial second-level analysis, with group (Tourette syndrome, control) as independent (between-subjects) factor, and task condition (Go, NoGo-correct, Choose-Go, Choose-NoGo and NoGo-error) as non-independent (repeated measures) factor. Three mean centred covariates modelled medication, ADHD diagnosis and OCD diagnosis $(1 / 0$ yes/no), thus removing potentially confounding variance from individual patient differences in medication status and comorbid ADHD and OCD symptoms.

F-contrasts were generated for all effects ('eye'), and group effects (controls versus Tourette syndrome) across all conditions and for Go, NoGo-correct, Choose-Go and Choose-NoGo individually. Post-hoc t-tests identified the direction of significant group effects. A conjunction analysis examined overlap of group differences in ChooseGo, Choose-NoGo and NoGo-correct. Task effect T-contrasts examined volitional action (Choose-Go $>$ Go), intentional inhibition (Choose-NoGo $>$ NoGo-correct), volitional action versus intentional inhibition (ChooseGo $>$ Choose-NoGo) and reactive inhibition (NoGocorrect $>$ Go), in controls and Tourette syndrome, respectively. Contrast estimate effect size plots for the five trial types were generated for the preSMA, bilateral insula and $M 1$, at the region's peak co-ordinate in the 'all effects' $F$ contrast, and for the IFG and caudate nucleus at the region's peak co-ordinate in the conjunction (IFG) and Choose-NoGo group difference (caudate nucleus).

A series of second-level models in Tourette syndrome participants examined correlations between task effects and Tourette syndrome symptom severity. One-sample $t$ tests of (i) Choose-Go and (ii) Choose-NoGo, with (i) YGTSS or (ii) PUTS as covariates, created an interaction between task effect and severity score. Medication and comorbidities were entered as covariates. T-contrasts tested for a positive correlation with YGTSS or PUTS.

Three further second-level models examined correlations between task effects relevant to inhibitory control (Choose-NoGo and NoGo-correct) and hyperactivity (Choose-Go) with OCD and ADHD symptom severity. Three one-sample $t$-tests of (i) Choose-Go, (ii) ChooseNoGo and (iii) NoGo-correct, with YBOCS and ASRS as covariates, created two interactions between task effect and severity score. Medication status was entered as a covariate. T-contrasts tested for a positive correlation with YBOCS or ASRS. Entering both severity scores to the models simultaneously enabled us to investigate the effects of OCD or ADHD severity while controlling for the other.

To verify that medication status did not affect univariate results, the subgroup of medicated patients $(n=9)$ was compared with the subgroup of unmedicated patients $(n=14)$ (Supplementary Methods).

Statistic images were thresholded at cluster-forming threshold $P<0.001$ for cluster-wise false-discovery rate (FDR) correction for multiple comparisons at $P<0.05$ (Chumbley et al., 2010; Eklund et al., 2016). Significant clusters were localized using the Anatomy toolbox (v2.2b, Eickhoff et al., 2007) and FSL Harvard-Oxford atlases (https://fsl.fmrib.ox.ac.uk/fsl/fslwiki/Atlases; accessed 30 November 2020).

\section{Psychophysiological interactions}

A series of psychophysiological interactions (PPIs) investigated how prefrontal cortex modulated activity elsewhere in the brain. We first examined whether preSMA or IFG was associated with changes in functional connectivity when choosing to go and choosing to withhold. Then, in participants with Tourette syndrome, we examined whether the strength of preSMA and IFG functional connectivity during Choose-Go and Choose-NoGo trials related to tic and premonitory sensation severity.

The first eigenvariate (weighted mean of BOLD timeseries) was extracted for the preSMA and IFG, by thresholding an F-contrast for all effects ('eye') at $P<1$ for each individual. A $10-\mathrm{mm}$ sphere was extracted at the preSMA peak in the second-level all effects $F$-contrast $(x 4, y 18$ and $z 48)$, and the IFG peak in the conjunction of group differences $(x 40, y 40$ and $\mathrm{z} 6)$. 
For each participant, a PPI term was calculated according to task effects (contrast weight: [1] for Choose-Go, and Choose-NoGo, trials respectively), and the time series of (i) preSMA and (ii) IFG. The Choose-Go and ChooseNoGo PPI terms for (i) preSMA and (ii) IFG were each entered to a first-level model, with regressors representing the region's BOLD activity (PPI.Y) and task effect (PPI.P). The three fMRI runs were concatenated (spm_concatenate.m), adding a constant (mean) column for each run. A 'block transitions' regressor modelled the transition from the end of one block to the start of the next. Six regressors modelled head movement, and for Tourette syndrome participants, a regressor of onsets and durations of tics identified in videos. $T$-contrasts were generated for the PPI term, and entered to second-level models.

Four second-level models examined the PPI of (i) preSMA and (ii) IFG, on Choose-Go and Choose-NoGo trials, in controls and Tourette syndrome participants (two-sample $t$-tests). F-contrasts and post-hoc T-contrasts tested for group effects (controls versus Tourette syndrome), and $T$-contrasts individual group effects.

In Tourette syndrome participants, four second-level models (one-sample $t$-tests) of (i) preSMA and (ii) IFG, on Choose-Go and Choose-NoGo trials, included YGTSS as a covariate; four further models included PUTS as a covariate; creating an interaction between PPI and severity score in all eight models. $T$-contrasts tested for a positive correlation with YGTSS or PUTS.

As in univariate analyses, PPI second-level models modelled medication, and ADHD/OCD diagnoses ( $1 / 0$ yes/ no), to remove potentially confounding variance from individual patient differences in medication status and comorbid ADHD and OCD symptoms; and contrasts were thresholded at $P<0.05$ FDR cluster-wise correction.

Again in Tourette syndrome participants, two further second-level models (one-sample $t$-tests) of (i) preSMA on Choose-Go trials and (ii) IFG on Choose-NoGo trials included YBOCS and ASRS as covariates, creating two interactions between PPI and severity score. Medication status was entered as a covariate. T-contrasts tested for a positive correlation with YBOCS or ASRS and were thresholded at $P<0.05$ FDR cluster-wise correction. Entering both severity scores to the models simultaneously enabled us to investigate the effects of $\mathrm{OCD}$ or $\mathrm{ADHD}$ severity while controlling for the other.

To verify that medication status did not affect PPI results, the subgroup of medicated patients $(n=9)$ was compared with the subgroup of unmedicated patients $(n=14)$ (Supplementary Methods).

Plots of the preSMA Choose-GoPPI correlation with PUTS were generated in SPM for the caudate nucleus, globus pallidus and thalamus, at each region's peak PPI coordinates, using adjusted data.

\section{Data availability}

Anonymized demographic, clinical and behavioural data; task code; JASP statistical analyses; tic regressor data and scripts; fMRI analysis scripts and second-level fMRI models are available at https://osf.io/94ybj (accessed 30 November 2020). Statistic images are in Neurovault (Gorgolewski et al., 2015) at https://neurovault.org/collec tions/9056/ (accessed 30 November 2020).

\section{Results}

\section{Intentional inhibition task}

Tourette syndrome participants did not choose to go or withhold more often than controls (\%Choose-Go controls: $53 \%$, Tourette syndrome: $56 \%, t=-0.924$, $P=0.361$ and $\left.\mathrm{BF}_{10}=0.420\right)$; nor did they make more NoGo errors (controls: $3 \%$, Tourette syndrome: $3 \%$, $t=-0.228, P=0.820$ and $\left.\mathrm{BF}_{10}=0.304\right)$. Tourette syndrome participants made slightly more Go omissions than controls (controls: 1\%, Tourette syndrome: $2 \%$, $t=-2.423, P=0.020$ and $\left.\mathrm{BF}_{10}=2.920\right)$, probably driven by one TS participant. Reaction times did not significantly differ between groups (Table 1 and Fig. 2).

There was no overall effect of face priming on $\%$ Choose-Go $\left(F=0.901, \quad P=0.410, \mathrm{BF}_{10}=0.162\right)$, no group difference $\left(F=0.886, P=0.352\right.$ and $\left.\mathrm{BF}_{10}=0.675\right)$, and no group by face prime type interaction $(F=0.610$, $P=0.546$ and $\mathrm{BF}_{10}=0.022$ ).

From the first block to the final block, the sample as a whole (not separated by group) chose to go more often on Choose trials $(+4 \%, F(1,42)=6.214, P=0.017$ and $\left.\mathrm{BF}_{10}=3.088\right)$; made more NoGo commission errors $\left(+2 \%, F(1,42)=7.491, P=0.009\right.$ and $\left.\mathrm{BF}_{10}=5.740\right)$; and were faster to respond on both Choose-Go $(-34 \mathrm{~ms}$, $F(1,42)=32.857, P<0.001$ and $\left.\mathrm{BF}_{10}=14989.073\right)$ and Go $\quad(-23 \mathrm{~ms}, \quad \mathrm{~F}(1,42)=18.687, \quad P<0.001 \quad$ and $\left.\mathrm{BF}_{10}=172.836\right)$ trials. There were no block effects on Go omissions $(+0.3 \%, F(1,42)=1.128, P=0.294$ and $\left.\mathrm{BF}_{10}=0.370\right)$ or NoGo error reaction time $(-24 \mathrm{~ms}$, $F(1,42)=0.023, P=0.882$ and $\left.\mathrm{BF}_{10}=0.355\right)$. This suggests that as the task progressed, participants as a whole became more impulsive responders, but did not decrease in attention to the task more generally, because they maintained their (high) rates of Go responses $198.5 \%$ in Block 1 versus $98.2 \%$ in Block 3). We next examined whether there were group effects, such as it would indicate that the patients became more impulsive as the experiment progressed than controls: there was no evidence to that effect, with no significant behaviour $\times$ group interactions in the six ANOVAs (\%Choose-Go: $F(1,42)=0.262, \quad P=0.611 \quad$ and $\quad \mathrm{BF}_{10}=0.520 ; \quad$ NoGo errors: $F(1,42)=0.178, P=0.675$ and $\mathrm{BF}_{10}=0.672$; Go omissions: $F(1,42)=0.511, P=0.479$ and $\mathrm{BF}_{10}=0.819$; Choose-Go RT: $\quad F(1,42)=0.054, \quad P=0.817 \quad$ and $\mathrm{BF}_{10}=3002.339$, large $\mathrm{BF}_{10}$ driven by main effect; NoGo error RT: $F(1,42)=0.068, P=0.799$ and $\mathrm{BF}_{10}=0.081$; Go RT: $F(1,42)=1.684, P=0.201, \mathrm{BF}_{10}=69.584$, large $\mathrm{BF}_{10}$ driven by main effect). This suggests that any neural 
A

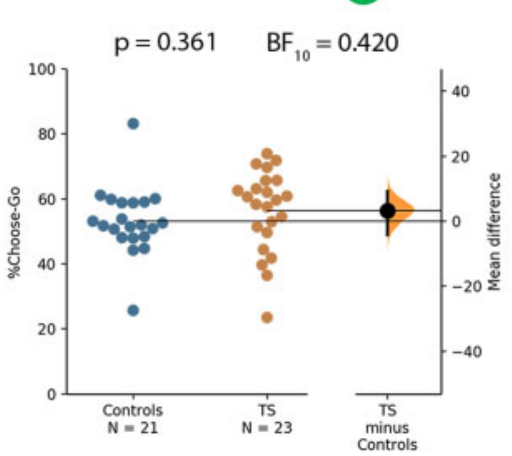

D

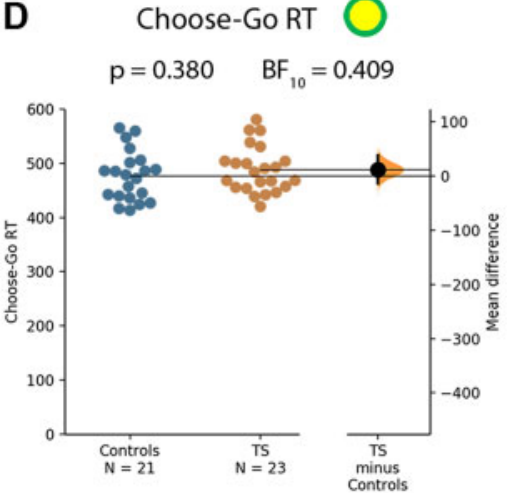

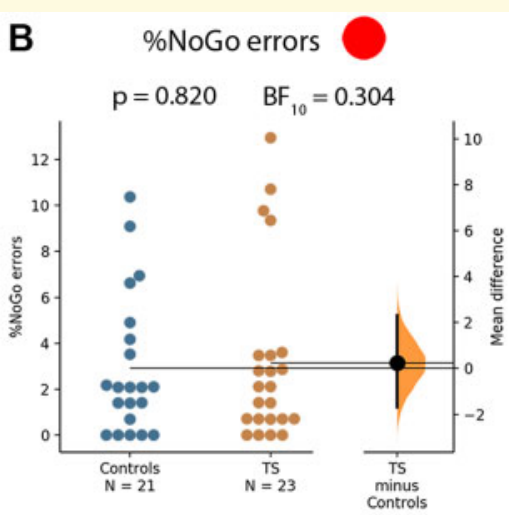

$\mathbf{E}$

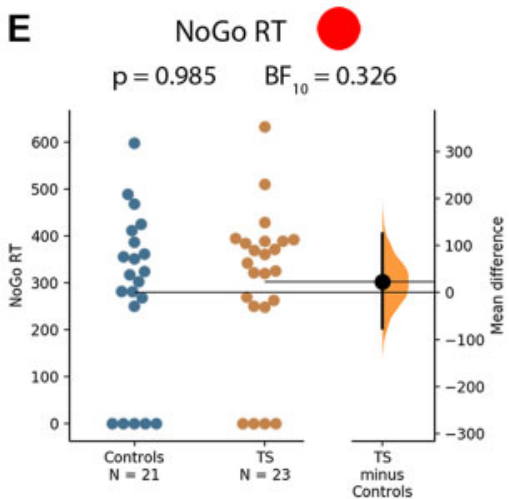

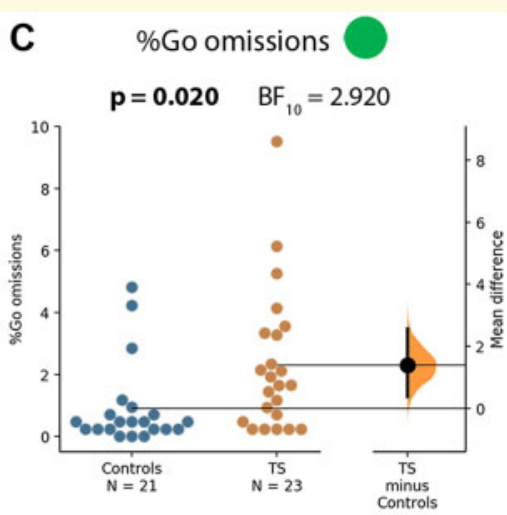
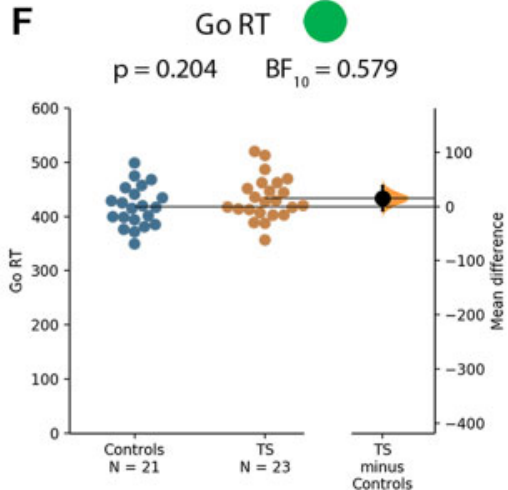

Figure 2 Behavioural performance on the intentional inhibition task, in control and Tourette syndrome participants. Data were visualized using estimation plots (www.estimationstats.com; accessed 30 November 2020, Ho et al., 2019).

group differences are not due to differential changes in attention to the task or changes in impulsivity as the experiment progressed.

\section{Face prime subliminality assessment}

Behavioural assessment of perception of the face primes suggested that some TS participants were able to detect the presence of a face, although they were unable to discriminate angry from neutral expressions (Supplementary Results).

\section{Univariate $\mathrm{fMRI}$ and contrast estimate effect sizes}

The F-contrast for all effects showed activity associated with Go, NoGo-correct, Choose-Go, Choose-NoGo and NoGo-error trials in controls and Tourette syndrome participants across prefrontal, parietal and insula cortices; visual cortices and cortical and subcortical motor regions (Fig. 3, Supplementary Table 2A).

Contrast estimate effect size plots at peak co-ordinates of the all effects $F$-contrast showed generally elevated activity in Tourette syndrome in preSMA, bilateral insula and M1 across trial types (Fig. 3). This was particularly notable during motor inhibition (Choose-NoGo and NoGo-correct), when primary motor cortex (M1) was suppressed (below 0) in controls but elevated in Tourette syndrome. However, elevated activity in Tourette syndrome did not necessarily pass stringent threshold criteria for significance when tested in the group effect wholebrain contrasts (Fig. 4). Contrast estimate effect size plots at peak co-ordinates of group differences (IFG, caudate nucleus), however, demonstrate the hyperactivity of these regions in Tourette syndrome.

There were significant group effects across all conditions (F-contrast), and for Choose-Go, Choose-NoGo and NoGo-correct (Supplementary Table 2D, F and H; Go group contrast not significant). Post-hoc t-tests confirmed that Tourette syndrome participants showed greater activity than controls across all conditions in bilateral IFG, right insula, caudate nucleus, putamen, globus pallidus and thalamus; during Choose-Go in right anterior IFG and sub-genual anterior cingulate cortex; during Choose-NoGo in bilateral anterior IFG and caudate nucleus and during NoGo-correct in right anterior IFG and left caudate nucleus (Fig. 4, Supplementary Table 2C, E, G and I). All T-contrasts for greater activity in controls than Tourette syndrome were not significant. A conjunction analysis of group difference overlap across Choose-Go, Choose-NoGo and NoGo-correct showed anterior IFG and ventromedial prefrontal cortex (Supplementary Table 2J). 


\section{A

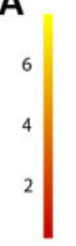

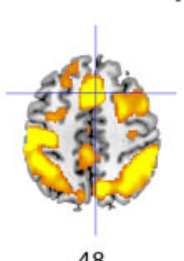

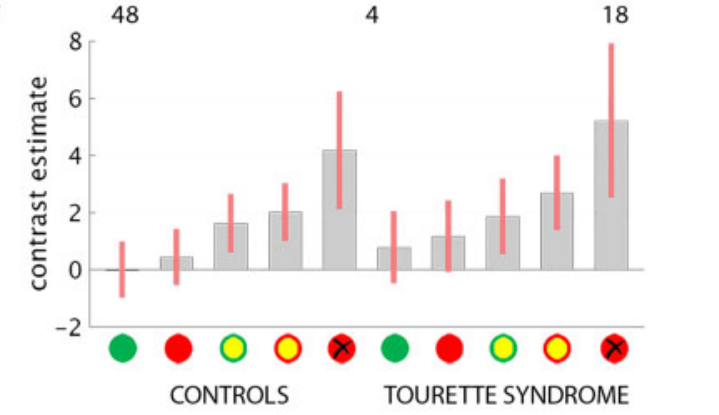

C

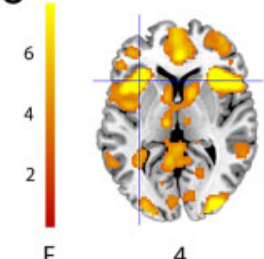

L insula $x-34$, y $20, z 4$

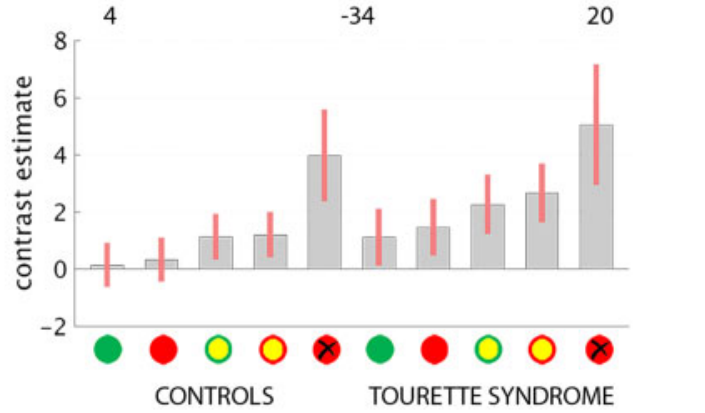

E

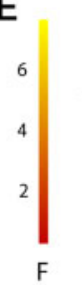

L caudate nucleus $x-12, y$ 18, $z-2$

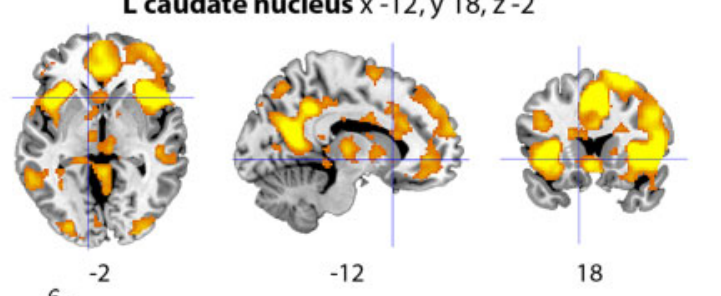

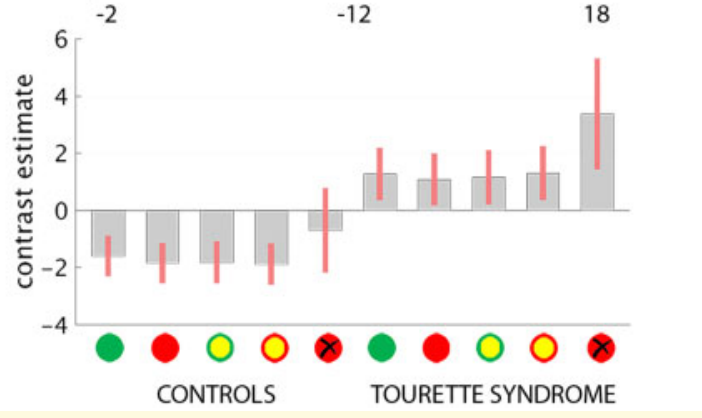

B

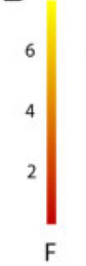

R IFG x 40, y 40, z 6

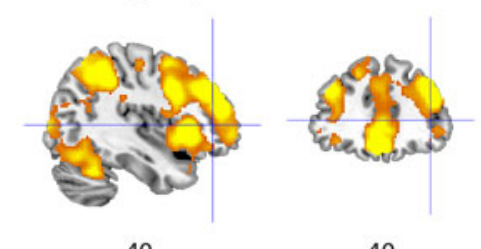

40

40

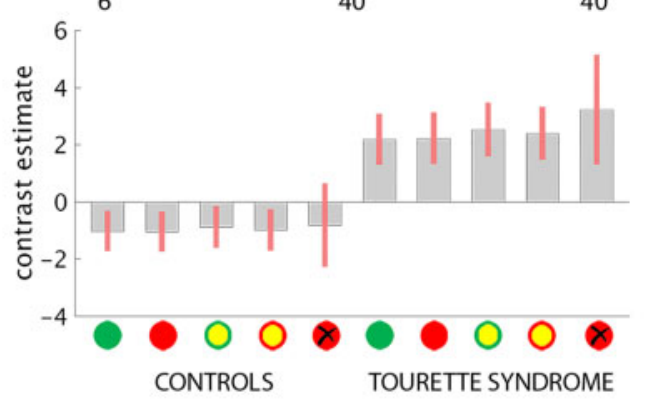

D

R insula $\times 34$, y 22, z 4
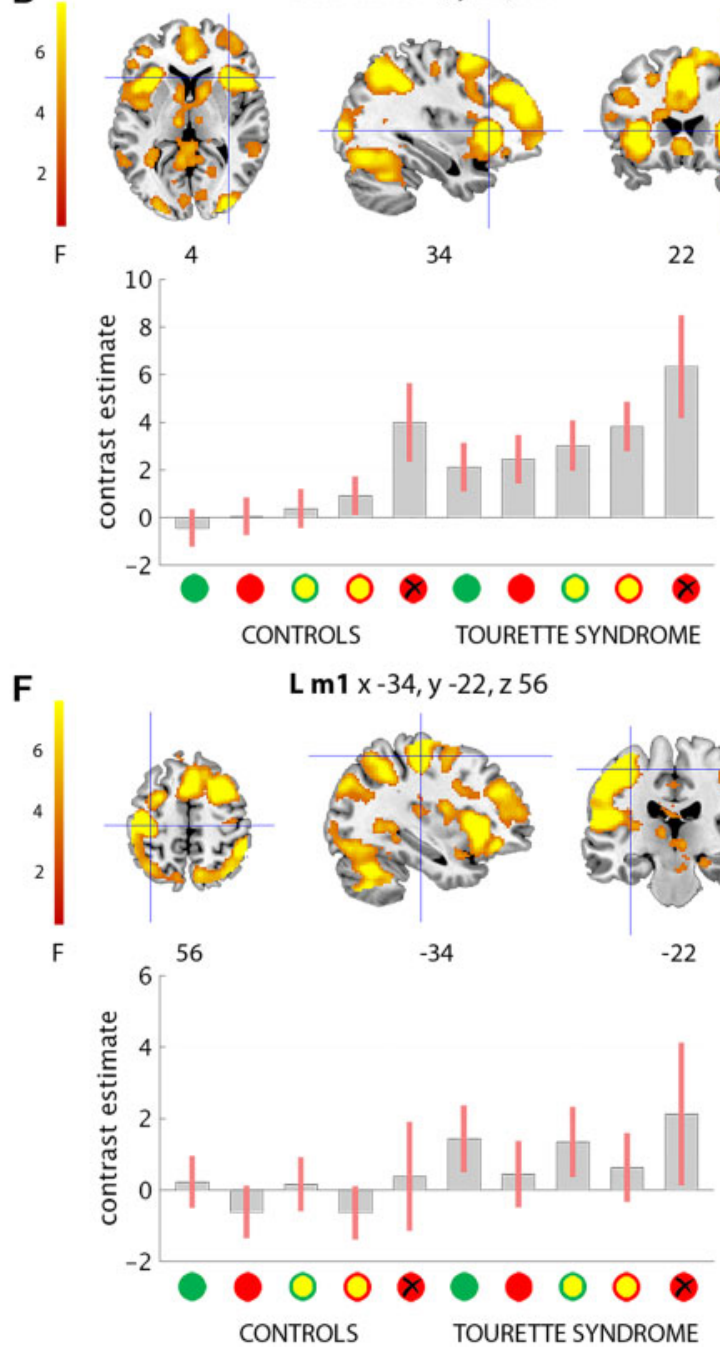

Figure 3 Contrast estimate effect size plots of activity during the intentional inhibition task. (A) preSMA, (B) right IFG, (C) left insula, (D) right insula, (E) left caudate nucleus, (F) left MI, for (left-to-right) controls (Go, NoGo-correct, Choose-Go, Choose-NoGo and NoGo-error) and Tourette syndrome (as for controls). Pink bar represents $90 \%$ confidence interval. Statistic image shown in (A-F) is the all effects ('eye') $F$ contrast. 
A
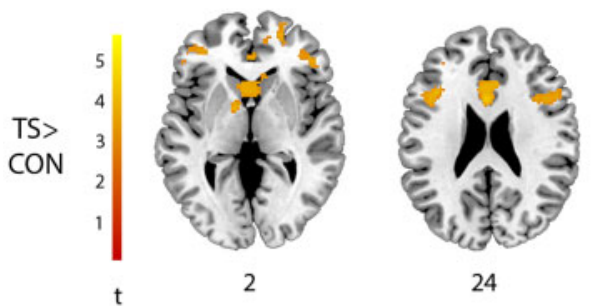

24

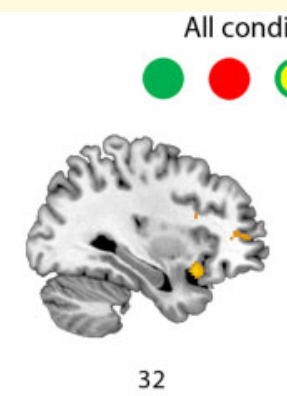

All conditions

B

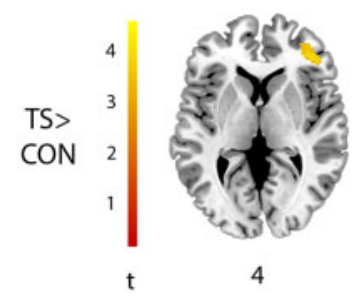

D

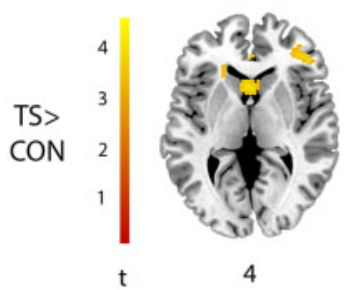

Choose-Go

O

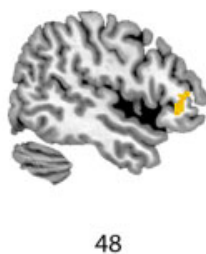

NoGo

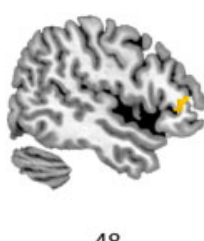

48

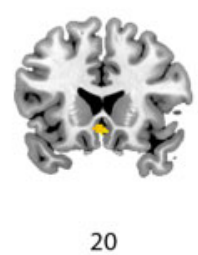

20

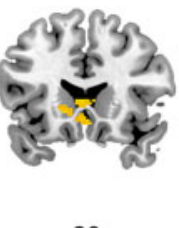

C

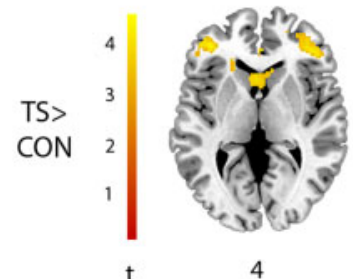

E

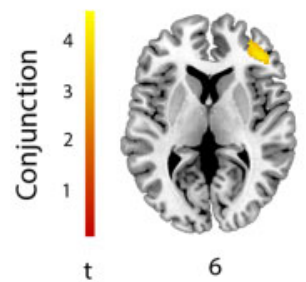

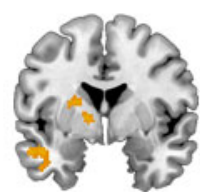

0

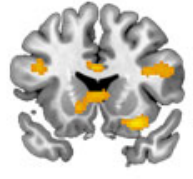

16
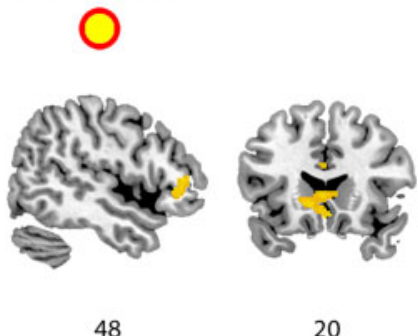

20

Figure 4 Group effects on the intentional inhibition task. Greater activity in Tourette syndrome participants (TS) than controls (CON) (A) across all conditions, and on (B) Choose-Go, (C) Choose-NoGo and (D) NoGo-correct trials; (E) conjunction of group difference overlap across (B), (C) and (D).

Task effect $T$-contrasts showed activity during volitional action (Choose-Go $>$ Go) in preSMA extending to the rostral cingulate zone, right IFG, bilateral insula and inferior parietal lobule in both controls and Tourette syndrome (Fig. 5A, Supplementary Table $2 \mathrm{~K}$ and L). In addition, there was activity in the thalamus in Tourette syndrome. During intentional inhibition (ChooseNoGo $>$ NoGo-correct), there was activity in the preSMA extending to the rostral cingulate zone, right IFG, bilateral insula and inferior parietal lobule in both groups (Fig. 5B, Supplementary Table $2 \mathrm{M}$ and N). Contrasting volitional action with intentional inhibition showed M1 in both groups, but to a greater anatomical extent and statistical height in controls (Fig. 5C, Supplementary Table $2 \mathrm{O}$ and $\mathrm{P}$ ). Finally, during reactive inhibition (NoGo-correct $>$ Go), there was activation of the left inferior frontal junction in both groups, and the right inferior frontal junction and right insula in controls, whereas Tourette syndrome participants showed additional activity in premotor cortex (Fig. 5D, Supplementary Table 2Q and R).
Second-level models in patients with Tourette syndrome tested for correlations between task effects (Choose-Go and Choose-NoGo) and Tourette syndrome symptom severity (YGTSS, PUTS). None were significant $(P<0.05$ FDR cluster-wise correction).

Three second-level models in Tourette syndrome participants that tested for correlations between task effects (Choose-Go, Choose-NoGo and NoGo-correct) and OCD or ADHD severity (YBOCS and ASRS) showed no significant effects $(P<0.05 \quad$ FDR cluster-wise correction).

\section{Psychophysiological interactions (PPI): preSMA and IFG}

Four second-level models examined changes in functional connectivity with (i) preSMA and (ii) IFG, according to psychological context of Choose-Go, and Choose-NoGo, in controls and Tourette syndrome.

In the preSMA PPI with Choose-Go, there was a significant effect of group (F-contrast, Supplementary Table 3A), 
A

Choose-Go > Go

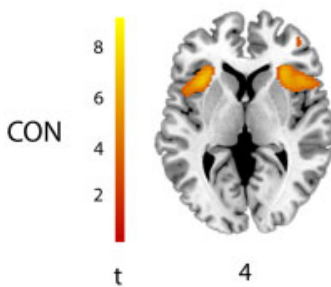

4

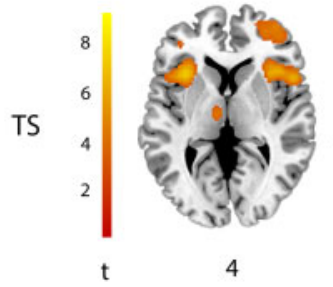

4

4
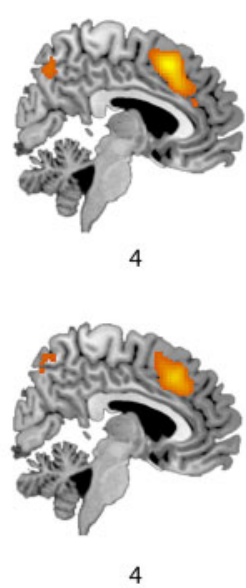

C

Choose-Go > Choose-NoGo
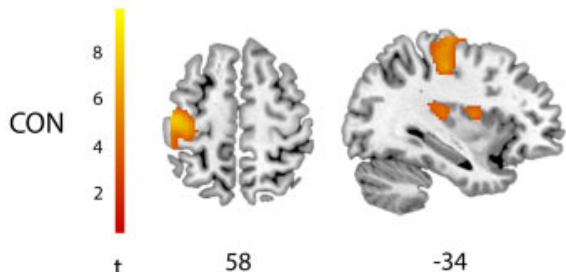

$-34$

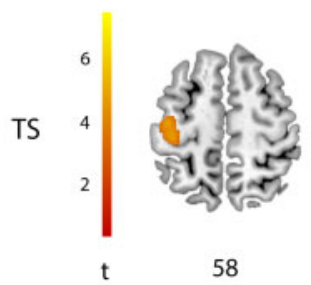

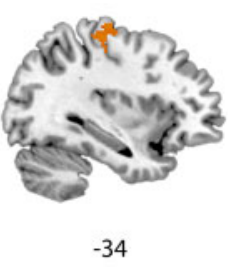

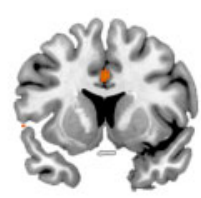

10

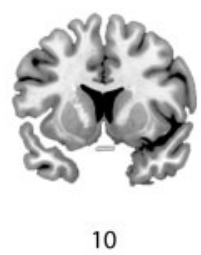

B
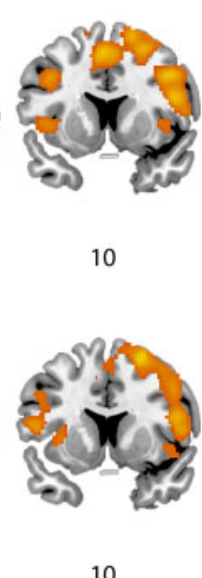

D
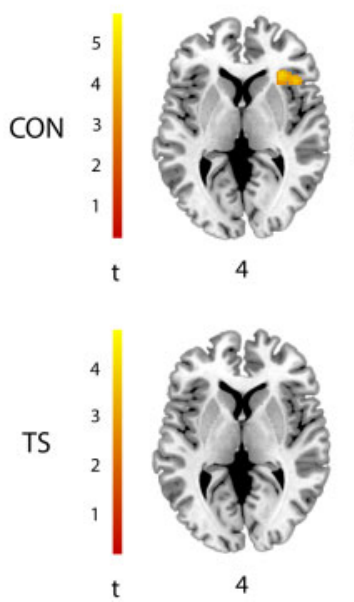
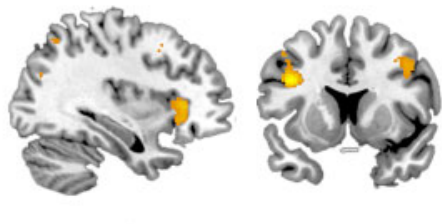

10

Choose-NoGo > NoGo

$\mathrm{O}$
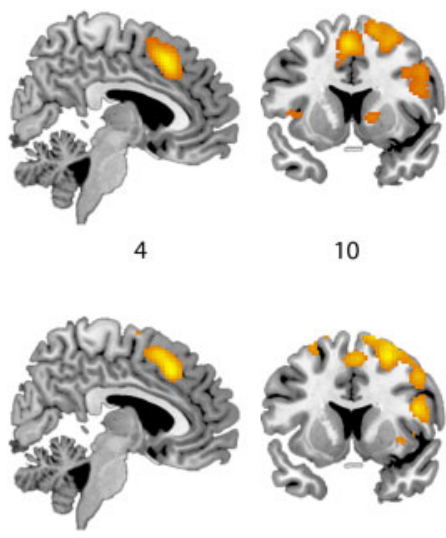

4

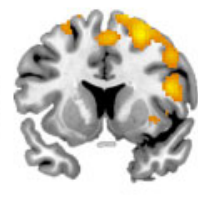

10

NoGo $>$ Go

34
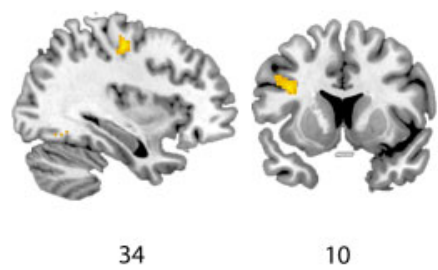

Figure 5 Task effects on the intentional inhibition task. Activity in controls (CON) and Tourette syndrome participants (TS) for (A) Choose-Go>Go, (B) Choose-NoGo >NoGo-correct, (C) Choose-Go>Choose-NoGo, and (D) NoGo-correct >Go.

which a post-hoc $t$-test revealed was due to greater taskinduced changes in functional connectivity between preSMA and the superior parietal lobule in Tourette syndrome than in controls (Fig. 6A, Supplementary Table 3B). The contrasts for individual group effects were not significant for either controls or Tourette syndrome. There were no significant effects for the preSMA PPI with Choose-NoGo.

In the IFG PPI with Choose-NoGo, there were no significant group effects. The contrast testing for a PPI in controls showed early visual cortices (Fig. 6B, Supplementary Table 3C); Tourette syndrome participants showed a PPI between the IFG and the frontal pole (Fig. 6C, Supplementary Table 3D). There were no significant effects for the IFG Choose-Go PPI.

\section{Psychophysiological interactions: Premonitory sensation severity (PUTS)}

In Tourette syndrome participants only, four PPI analyses tested whether preSMA and IFG connectivity during Choose-Go and Choose-NoGo trials varied in relation to premonitory sensation severity from PUTS scores. There were no regions where functional connectivity of the IFG varied in proportion to premonitory sensation severity. However, the preSMA PPI showed a significant correlation with PUTS in the caudate nucleus, globus pallidus and thalamus during Choose-Go (Fig. 7A-D, Supplementary Table 3E). The 
A

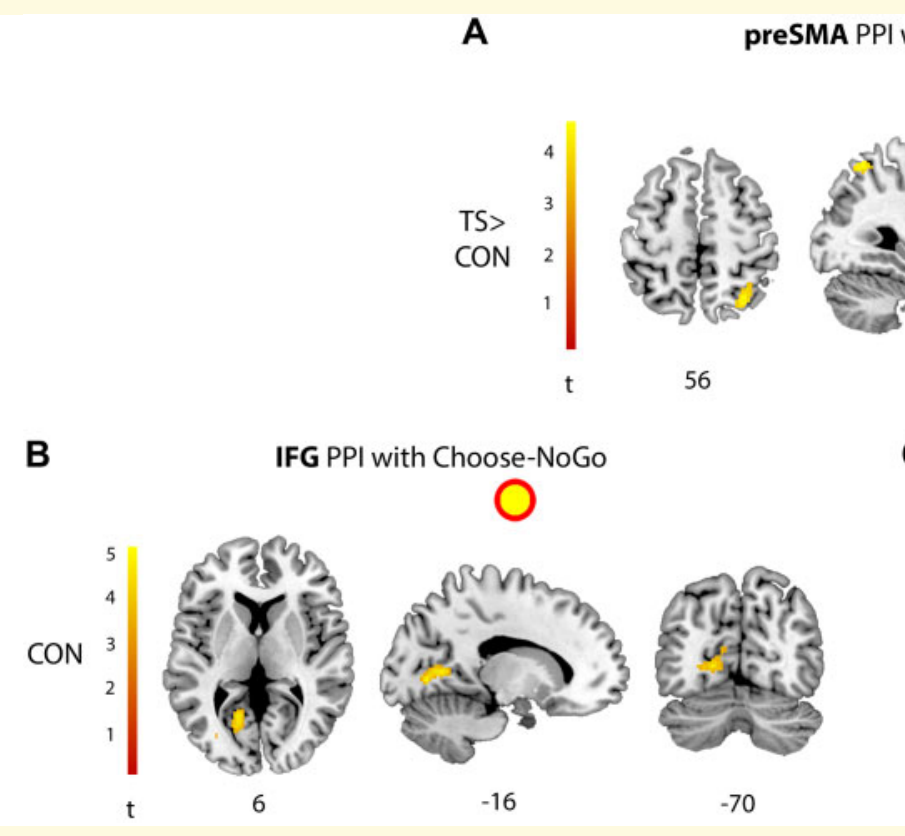

C

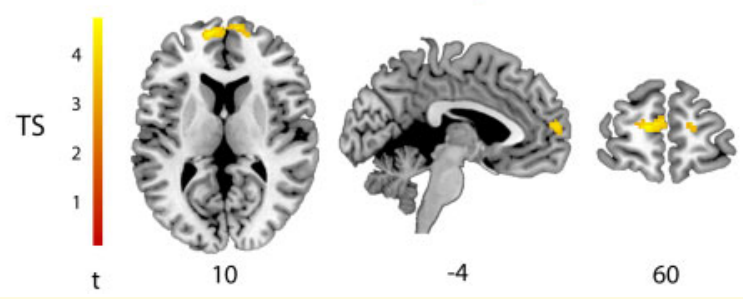

Figure 6 Group PPI results. (A) Greater PPI in Tourette syndrome participants (TS) than controls (CON) from preSMA during Choose-Go. IFG PPI with Choose-NoGo in (B) controls and (C) Tourette syndrome.

preSMA Choose-NoGo PPI with PUTS was not significant.

\section{Psychophysiological interactions: Tic severity (YGTSS)}

In Tourette syndrome participants only, four PPI analyses examined whether preSMA and IFG connectivity during Choose-Go and Choose-NoGo trials varied in relation to tic severity according to YGTSS scores. There were no regions where functional connectivity of the preSMA varied in proportion to tic severity. However, the IFG PPI showed a significant correlation with YGTSS in early visual cortices and V4 during Choose-Go (Fig. 7E, Supplementary Table 3F). The IFG Choose-NoGo PPI with YGTSS was not significant.

\section{Psychophysiological interactions: OCD and ADHD severity}

In Tourette syndrome participants only, two PPI analyses examined whether preSMA connectivity during Choose-Go trials, and IFG connectivity during Choose-NoGo trials, varied in relation to OCD severity (YBOCS) or ADHD severity (ASRS). There were no regions where functional connectivity varied in proportion to OCD severity. However, the IFG PPI showed a significant correlation with ASRS in premotor cortex (Fig. 7F, Supplementary Table 3G). The preSMA PPI with ASRS was not significant.

\section{Discussion}

Tourette syndrome is characterized by both the 'unvoluntary' nature of tics, and the ability of many patients to intentionally supress tic expression. To uncover the neural mechanisms by which people with Tourette syndrome control action, we employed an intentional inhibition task, in which participants chose whether to execute or withhold a simple movement. By monitoring patients' tics, we could remove confounding influences of tic expression or suppression on our fMRI measurements. This enabled us to undertake a comprehensive analysis of the interactions between prefrontal and motor regions underpinning control of voluntary action in Tourette syndrome in comparison to a control group without tics.

We found that the neural processes by which Tourette syndrome participants choose to act, or withhold movements, are anatomically similar to controls, encompassing activity in cardinal prefrontal and motor regions including the preSMA. We uncovered further subtleties in these network operations in Tourette syndrome, observing heightened activity in primary motor cortex-even when no action is made-and significantly greater activity than controls in anterior IFG and caudate nucleus when choosing to go, choosing to withhold and on NoGo trials that captured reactive inhibition. Functional connectivity analyses further elucidated the impact of individual differences in symptom severity. When choosing to go, patients with worse premonitory sensations showed increased connectivity between preSMA and the subcortical nuclei thought critical for tic genesis, highlighting a neural 
A

preSMA PPI with Choose-Go: +correlation with PUTS

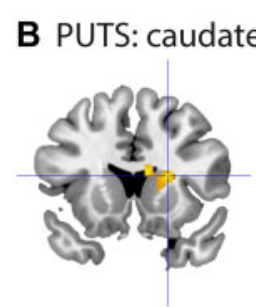

16
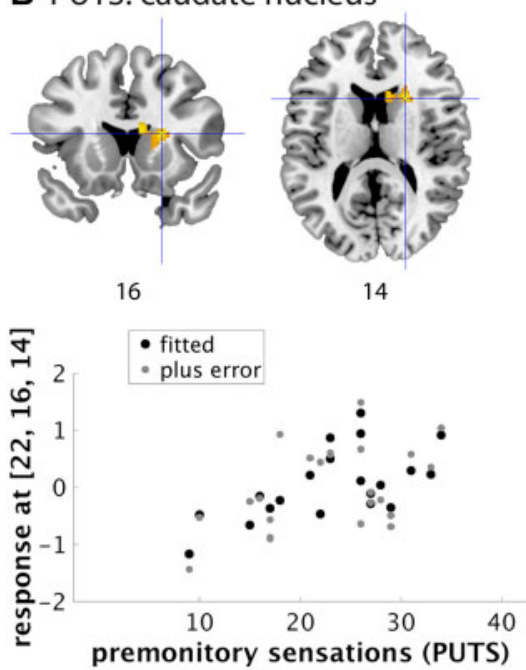

14

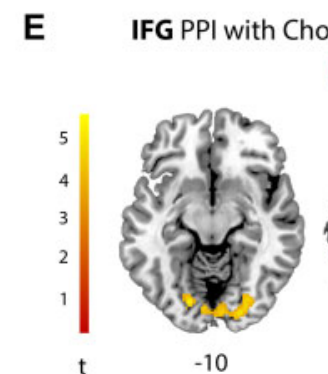

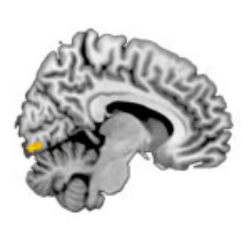

8

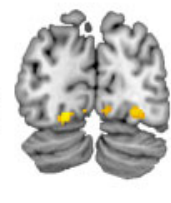

$-80$

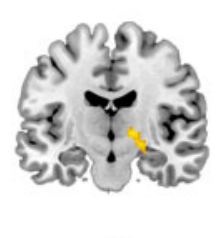

$-14$

C PUTS: globus pallidus
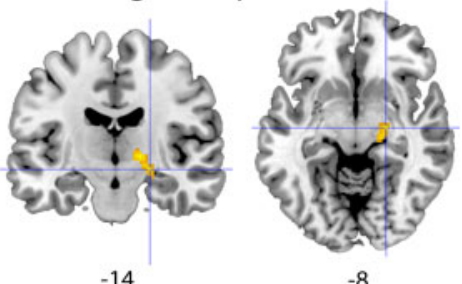

$-8$

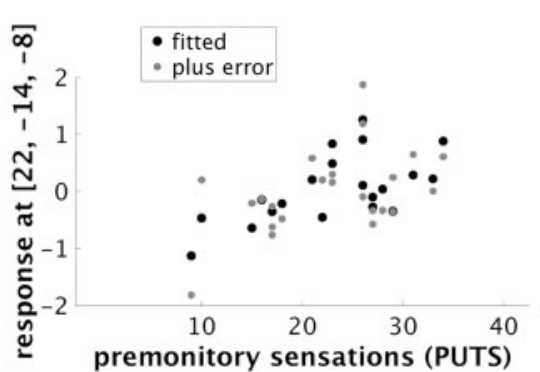

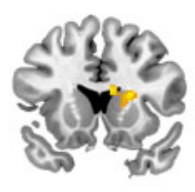

16

Figure 7 Regions showing a correlation between PPI functional connectivity and premonitory sensations (PUTS), tic severity (YGTSS) and ADHD severity (ASRS). The worse the premonitory sensations, tic severity or ADHD severity the greater the functional connectivity. (A) preSMA functional connectivity with PUTS during Choose-Go: caudate nucleus, globus pallidus, thalamus; (B) to (D): correlation plots of preSMA Choose-Go PPI with PUTS in (B) caudate nucleus, (C) globus pallidus, (D) thalamus; (E) right IFG functional connectivity with YGTSS during Choose-Go: early visual cortices and V4; (F) right IFG functional connectivity with ASRS during Choose-NoGo: premotor cortex.

cascade by which stronger premonitory sensations may intensify the urge to move.

Together, these results suggest that the neural processes for action control in people with Tourette syndrome are anatomically similar to those used by people without tics when choosing to withhold actions. However, in Tourette syndrome, these processes operate against a backdrop of basal ganglia dysconnectivity and elevated primary motor cortex reactivity. The result is that greater prefrontal leverage is required to

F IFG PPI with Choose-NoGo: +correlation with ASRS
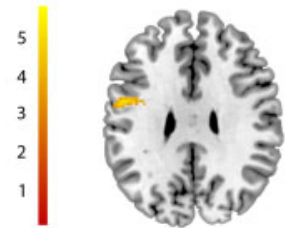

30

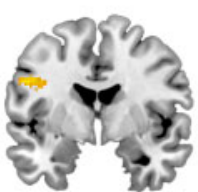

$-2$

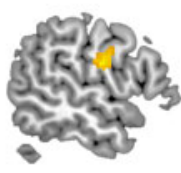

$-56$
D PUTS: thalamus
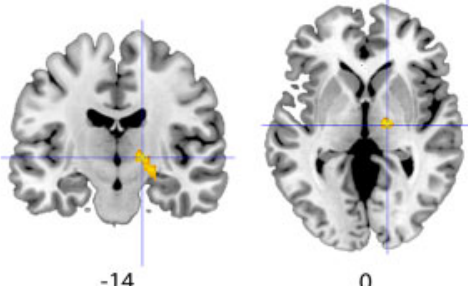

0

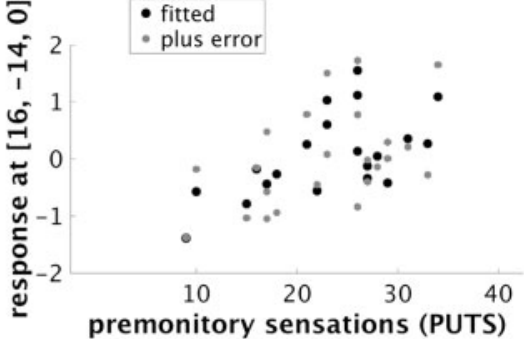


prefrontal motor control network supporting multiple forms of action control is perhaps unsurprising, given its evolutionary efficiency (Friston and Price, 2003), and the wide spectrum of action choice types from 'internally cued' to 'externally cued' or 'what-when-whether' categories (Nachev et al., 2008; Passingham et al., 2010; Zapparoli et al., 2017a). Where participants with Tourette syndrome differed from controls were in the level of activity within these cardinal motor control networks.

In Tourette syndrome participants, activity was generally elevated across several regions, including the IFG, right insula, basal ganglia (caudate nucleus, putamen and globus pallidus) and thalamus. On trials specifically involving motor inhibition, anterior IFG and caudate nucleus were significantly hyperactive compared to controls. A more posterior IFG site (pars opercularis) is commonly associated with reactive motor inhibition, for example, on the stop signal task (Aron et al., 2004; Rae et al., 2015). However, anterior IFG was linked to tic suppression in a previous study comparing suppression to 'free ticcing' (Ganos et al., 2014a). Meta-analyses of reactive inhibition also reveal multiple clusters of IFG activity along the extent of the gyrus (Rae et al., 2014; Guo et al., 2018). It is also notable that multiple cognitive domains beyond motor inhibition are associated with IFG function, and relevant to symptoms of Tourette syndrome, such as vocalization and stimulus salience processing (Amunts and Zilles, 2012; Hampshire and Sharp, 2015) although these were not explicitly manipulated in this study. Together, these data suggest that IFG, including more anterior segments, is hyperactive in Tourette syndrome, and likely underpins volitional withholding of tics and non-tic actions. Compared to controls, greater activity is required to overcome subcortical and primary motor cortex circuitry tipped towards a state of motor execution.

Further evidence for a heightened state of motor excitability in Tourette syndrome was obtained by examining primary motor cortex. Here, the effect size plots showed that when controls inhibited actions (on both NoGo and Choose-NoGo trials) primary motor cortex activity was suppressed. In contrast, primary motor cortex activity was not suppressed in participants with Tourette syndrome (mean contrast estimates were above zero even though participants were not moving). This finding cannot be attributed to tic expression, which was controlled for within the analytic models. Moreover, tic expression would have affected the implicit baseline (inter-trial intervals) against which task events and hence NoGo and Choose-NoGo contrasts were computed. This intriguing finding of elevated primary motor cortex activity in Tourette syndrome extends transcranial magnetic stimulation data, showing heightened primary motor cortex excitability in Tourette syndrome during NoGo states (Draper et al., 2015), and greater reduction in primary motor cortex excitability during tic suppression in patients best able to withhold tics (Ganos et al., 2018a).
These data also support the hypothesis that tonic regulation of excitability within motor pathways may underlie remission of tics in adolescents whose tics reduce with age (Jackson et al., 2015), whereas heightened motor cortex excitability remains in those who express tics into adulthood.

Pre-supplementary motor area activity was not significantly different between Tourette syndrome and control participants when choosing whether to act or withhold. The pre-supplementary motor area is a principal site of voluntary action; electrical stimulation here elicits the urge to move (Fried et al., 1991) and focal activation underscores 'what-when-whether' decisions during fMRI (Zapparoli et al., 2017a). Curiously, prior fMRI studies have shown SMA (rather than preSMA) activity prior to release of tics (Bohlhalter et al., 2006; Neuner et al., 2014): We proposed that the role of the preSMA in tics may be ascribing a 'somewhat intended' or 'unvoluntary' experience to 'explain away' motor prediction errors, arising from the release of tics fostered by SMA and basal ganglia dysfunction (Rae et al., 2019). We argue that during tic suppression, the preSMA may signal to subcortical structures, in particular the sub-thalamic nucleus, to pause motor outflow, whereas the IFG amplifies this inhibitory effect (Rae et al., 2015, 2019). Both these propositions imply that the preSMA is not a site of overt dysfunction in Tourette syndrome relative to controls, whereas basal ganglia and lateral prefrontal sites are implicated in tic genesis and suppression, respectively. Meta-analyses show that across tasks, both IFG and SMA, but not preSMA, are hyperactive in Tourette syndrome (Polyanska et al., 2017). Future application of multivariate pattern analysis techniques (Haxby et al., 2014) holds potential to determine whether sub-populations of preSMA neurons underpinning choices to move or withhold (Fedota et al., 2014) are functionally different in patients with Tourette syndrome. More finegrained neuroimaging (at higher field strength than employed here) will be valuable in exploring interactions between IFG and the sub-thalamic nucleus. This may delineate more precisely how hyperactivity within IFG and caudate nucleus contributes to pausing of basal ganglia outflow to primary motor cortex.

\section{Psychophysiological interactions}

We used functional connectivity analyses to explore how prefrontal and motor planning regions, namely the IFG and preSMA, interact with downstream regions, including basal ganglia. Greater functional connectivity was observed from the preSMA during Choose-Go (but not Choose-NoGo) trials, and from the IFG during ChooseNoGo (but not Choose-Go) trials. These results indicate that the preSMA perhaps makes a stronger contribution to movement production and the IFG a stronger contribution to movement withholding (Aron et al., 2016). Next, we examined how preSMA and IFG interactions 
scale according to disorder severity in Tourette syndrome. Notably, when choosing to go, preSMA functional connectivity to the caudate nucleus, globus pallidus and thalamus was stronger in patients with worse premonitory sensations. Thus, pathways driving volitional production of movement appear hyper-connected in individuals with greater premonitory sensations, which may be underscored by structural connectivity of white matter tracts connecting preSMA to the basal ganglia (Worbe et al., 2015).

We did not observe increased preSMA functional connectivity with the insula, a region implicated in generating premonitory sensations that can foster tic production through outputs to midline motor regions (Jackson et al., 2011; Cavanna et al., 2017; Conceicao et al., 2017). Speculatively, this may reflect greater insular inputs to the SMA, rather than preSMA (Rae et al., 2019). Also, our task modelled 'whether' decisions to release or withhold movements, and correspondingly elicited activity in canonical voluntary action regions. However, this arguably has different ecological validity compared to blink suppression tasks (Mazzone et al., 2010; van der Salm et al., 2018), which might engender stronger feelings of urge and premonitory sensations.

There were a few relationships identified for IFG connectivity, in terms of tic severity (visual cortices) and ADHD severity (premotor cortex). The fact that higher severity of ADHD symptoms was associated with greater connectivity between the IFG and premotor cortex when choosing to inhibit suggests that stronger leverage of prefrontal resources to motor preparation cortices for the volitional withholding of action may be required in patients with worse ADHD (regardless of diagnostic status). We did not observe any relationships between univariate task effects or connectivity with OCD severity, suggesting that severity of obsessive compulsive symptoms did not affect the main findings of IFG and striatal hyperactivity in the patient group compared to controls (nor OCD diagnostic status, which was entered as a covariate in group analyses). Employing symptom severity scales alongside recording diagnostic status can be useful in order to distinguish overall clinical cohort effects from effects of within-group heterogeneity.

\section{Subliminal face perception in TS}

Our paradigm included an exploratory 'face priming' element, in which motor cues were preceded by brief (16 ms) presentations of neutral, angry or scrambled faces. Social context clearly influences tic expression, provoking echophenomena or exacerbating tic expression through social scrutiny (Eapen et al., 1994; Ganos et al., 2012). We previously found that supraliminal (i.e. consciously perceived) face stimuli-portraying neutral or angry expressions-evoke insula hyperactivity in Tourette syndrome, and further, that insula to basal ganglia functional connectivity scaled with the severity of premonitory sensations (Rae et al., 2018). This suggests that the insula is a tic trigger site, cueing motor responses to affective stimuli. However, here we observed no effect of masked face primes on how frequently participants chose to act; nor significant group differences. We therefore collapsed across face prime types in fMRI analyses to increase statistical power. Although we intended the face primes to be subliminal (unconscious), our subsequent detection checks found that patients with Tourette syndrome detected their presence, despite the forward-and-backward masking. This heightened perceptual ability may represent a core feature of Tourette syndrome, or alternatively may arise experientially after years of often uncomfortable social scrutiny.

\section{Study limitations and future directions}

We selected the intentional inhibition task as an exemplary paradigm for measuring voluntary action, voluntary inhibition and reactive inhibition within the same experimental session. This is distinct from tic suppression studies, for which a direct comparison task to control participants is not possible. Blink suppression paradigms bridge this gap, in addition to capturing the urge nature of both tics and blinks, but generate different numbers of trials, and unbalance data across individuals and groups, impacting statistical power. Hence, the present task offers a purer index of voluntary action control for group comparisons. It may be valuable for future investigations to incorporate all three types of task within a single study, enabling evaluation across dimensions of naturalistic urge with equivalent information sampling between participants.

Several patient participants reported feeling fatigued after completion of the study. Therefore, we checked for possible differences in attention to the task in the patients versus controls, in case neural differences observed in Tourette syndrome were due to differential changes in behaviour as the experiment progressed. From the first to the final block, participants as a whole chose to go more often, made more NoGo commission errors, and were faster to respond on Choose-Go and Go trials, but maintained their high rates of Go responses. This suggests that as the task progressed, participants became more impulsive responders, but did not decrease in their attention to the task for generally. Although there were significant block effects on impulsivity, the numerical changes were not vast: thus the changes in impulsive responding in the sample as a whole were subtle, but significant. However, there were no group by time interactions, such that the patients did not become more impulsive as the experiment progressed than controls. This gives confidence that the neural hyperactivity seen in the Tourette syndrome participants is not due to differential changes in attention to the task or changes in impulsivity. 
Deployment of an intentional inhibition task requires that participants are not simultaneously attempting to suppress tics since this would impact fMRI measurements of motor inhibition networks. Therefore, we allowed participants to tic and applied the alternative strategy of using video recordings of participants' face and limbs to generate 'tic regressors' and thus remove the influence of tic expression on fMRI analyses (Neuner et al., 2007; Thomalla et al., 2014; Rae et al., 2018). Although this approach was as comprehensive as conceivably feasible, it remains possible that some phonic tics were not captured. Following Thomalla et al. (2014), we monitored the live video feeds to note observed tics in the first instance, and then conducted a thorough offline tic rating procedure, incorporating multiple raters, to ensure optimum fidelity of the tic timelines that were entered to fMRI statistical models. Such additional challenges are important to consider when studying movement disorder populations who may be expressing symptoms during scanning, especially hyperkinesias.

We accounted for comorbidities and medications by including these as covariates in statistical analyses. The 'TS spectrum' ranges from 'pure TS', characterized by simple tics alone, to 'full-blown TS' in which the motor symptoms are accompanied by complex tic expressions (such as echo- and coprophenomena) and multiple comorbidities, often including ADHD, autism and OCD (Robertson and Eapen, 2014; Martino et al., 2017). In a mixed sample, it may be difficult to disentangle whether prefrontal hyperactivation (for example) is inherently due to Tourette syndrome, or related to a comorbid neurodevelopmental disorder also involving frontostriatal circuits. However, we feel studying a cohort that spans the full 'TS spectrum' is essential since it is likely 'not a unitary condition' (Robertson, 2015). Using covariates is one approach to tackle confounding influences of ADHD and OCD while, in larger samples, patient stratification into sub-groups would enable greater insight.

Decisions on how to model and analyse fMRI data, including how to control for comorbidities and investigate the impacts of symptom severity, all influence researcher degrees of freedom. Given the number of possible analytic permutations in neuroimaging studies, these can be very large. One useful way to limit such degrees of freedom is to pre-register an fMRI analysis plan before the data are observed and/or analysed. If we had done so for our study, we could have formally defined in advance (for example) when compensatory neural processes to evoke similar behavioural performance to controls, such as we observed with the IFG, were expected. This enables one to demonstrate effects via 'confirmatory' tests. If not formally specified in advance via a pre-registered plan, analyses can be conceptualized as exploratory tests instead.

Modelling behavioural data, as well as neural activity, can bring useful insights, especially if model parameters are then reapplied to neuroimaging analyses. Such methods have yet to be widely applied in Tourette syndrome (Maia and Conceicao, 2017). Drift diffusion modelling offers one promising approach to understand how the motor system is tipped towards cortical excitability, and modulated under reactive motor inhibition (Draper et al., 2015) and intentional tic suppression (Ganos et al., 2018a). Voluntary decisions to move or withhold can be explained by accumulation of activity to motor thresholds (Sebastian et al., 2018). Altered thresholds or accumulation rates can differentiate other patient groups, including Parkinsonian sub-types (Zhang et al., 2016). It is plausible that patients with worse premonitory sensations or tic severity are distinguished by lower thresholds and faster accumulation rates for choices to go, and that such parameters correlate with activity in cortico-striato-thalamo-cortical circuitry.

\section{Conclusion}

People with and without Tourette syndrome use similar neuroanatomical architecture in the release and intentional withholding of actions. However, greater prefrontal engagement is required in Tourette syndrome to prevent release of movements arising from hyperactivity within downstream motor regions, notably primary motor cortex. Midline motor regions typically associated with voluntary action interact with the basal ganglia in proportion to the severity of premonitory sensations, highlighting how individuals with greater premonitory urges experience hyper-connectivity of networks that underpin volitional movement.

\section{Supplementary material}

Supplementary material is available at Brain Communications online.

\section{Funding}

This work was funded by a donation from the Dr. Mortimer and Theresa Sackler Foundation.

\section{Competing interests}

The authors report no competing interests.

\section{References}

Amunts K, Zilles K. Architecture and organizational principles of Broca's region. Trends Cogn Sci 2012; 16: 418-26.

Aron AR, Herz DM, Brown P, Forstmann BU, Zaghloul K. Frontosubthalamic circuits for control of action and cognition. J Neurosci 2016; 36: 11489-95. 
Aron AR, Robbins TW, Poldrack RA. Inhibition and the right inferior frontal cortex. Trends Cogn Sci 2004; 8: 170-7.

Bohlhalter S, Goldfine A, Matteson S, Garraux G, Hanakawa T, Kansaku K, et al. Neural correlates of tic generation in Tourette syndrome: an event-related functional MRI study. Brain 2006; 129: 2029-37.

Brass M, Haggard P. The hidden side of intentional action: the role of the anterior insular cortex. Brain Struct Funct 2010; 214: 603-10.

Brass M, Haggard P. The what, when, whether model of intentional action. Neuroscientist 2008; 14: 319-25.

Cavanna AE, Black KJ, Hallett M, Voon V. Neurobiology of the premonitory urge in Tourette's syndrome: pathophysiology and treatment implications. J Neuropsychiatr Clin Neurosci 2017; 29: 95-104.

Cavanna AE, Nani A. Tourette syndrome and consciousness of action. Tremor Other Hyperkinet Mov 2013; 3: 3.

Chumbley J, Worsley K, Flandin G, Friston K. Topological FDR for neuroimaging. Neuroimage 2010; 49: 3057-64.

Conceicao VA, Dias A, Farinha AC, Maia TV. Premonitory urges and tics in Tourette syndrome: computational mechanisms and neural correlates. Curr Opin Neurobiol 2017; 46: 187-99.

Draper A, Jude L, Jackson GM, Jackson SR. Motor excitability during movement preparation in Tourette syndrome. J Neuropsychol 2015; 9: 33-44.

Eapen V, Moriarty J, Robertson MM. Stimulus induced behaviours in Tourette's syndrome. J Neurol Neurosurg Psychiatr 1994; 57: 853-5.

Eickhoff SB, Paus T, Caspers S, Grosbras MH, Evans AC, Zilles K, et al. Assignment of functional activations to probabilistic cytoarchitectonic areas revisited. Neuroimage 2007; 36: 511-21.

Eklund A, Nichols TE, Knutsson H. Cluster failure: why fMRI inferences for spatial extent have inflated false-positive rates. Proc Natl Acad Sci USA 2016; 113: 7900-5.

Fedota JR, Hardee JE, Perez-Edgar K, Thompson JC. Representation of response alternatives in human presupplementary motor area: multi-voxel pattern analysis in a go/no-go task. Neuropsychologia 2014; 56: 110-8.

Fried I, Katz A, McCarthy G, Sass KJ, Williamson P, Spencer SS, et al. Functional organization of human supplementary motor cortex studied by electrical stimulation. J Neurosci 1991; 11: 3656-66.

Friston KJ, Price CJ. Degeneracy and redundancy in cognitive anatomy. Trends Cogn Sci 2003; 7: 151-2.

Frundt O, Woods D, Ganos C. Behavioral therapy for Tourette syndrome and chronic tic disorders. Neurol Clin Pract 2017; 7: 148-56.

Ganos C. Tics and Tourette's: update on pathophysiology and tic control. Curr Opin Neurol 2016; 29: 513-8.

Ganos C, Kahl U, Brandt V, Schunke O, Baumer T, Thomalla G, et al. The neural correlates of tic inhibition in Gilles de la Tourette syndrome. Neuropsychologia 2014a; 65: 297-301.

Ganos C, Kuhn S, Kahl U, Schunke O, Feldheim J, Gerloff C, et al. Action inhibition in tourette syndrome. Mov Disord 2014b; 29: 1532-8.

Ganos C, Ogrzal T, Schnitzler A, Munchau A. The pathophysiology of echopraxia/echolalia: relevance to Gilles de la Tourette syndrome. Mov Disord 2012; 27: 1222-9.

Ganos C, Rocchi L, Latorre A, Hockey L, Palmer C, Joyce EM, et al. Motor cortical excitability during voluntary inhibition of involuntary tic movements. Mov Disord 2018a; 33: 1804-9.

Ganos C, Rothwell J, Haggard P. Voluntary inhibitory motor control over involuntary tic movements. Mov Disord 2018b; 33: 937-46.

Goodman WK, Price LH, Rasmussen SA, Mazure C, Fleischmann RL, Hill CL, et al. The Yale-Brown Obsessive Compulsive Scale. I. Development, use, and reliability. Arch Gen Psychiatr 1989; 46: 1006-11.

Gorgolewski KJ, Varoquaux G, Rivera G, Schwarz Y, Ghosh SS, Maumet C, et al. NeuroVault.org: a web-based repository for collecting and sharing unthresholded statistical maps of the human brain. Front Neuroinform 2015; 9: 8 .

Guo Y, Schmitz TW, Mur M, Ferreira CS, Anderson MC. A supramodal role of the basal ganglia in memory and motor inhibition: Metaanalytic evidence. Neuropsychologia 2018; 108: 117-34.

Hampshire A, Sharp DJ. Contrasting network and modular perspectives on inhibitory control. Trends Cogn Sci 2015; 19: 445-52.

Haxby JV, Connolly AC, Guntupalli JS. Decoding neural representational spaces using multivariate pattern analysis. Annu Rev Neurosci 2014; 37: 435-56.

Ho J, Tumkaya T, Aryal S, Choi H, Claridge-Chang A. Moving beyond P values: data analysis with estimation graphics. Nat Methods 2019; 16: 565-6.

Jackson GM, Draper A, Dyke K, Pepes SE, Jackson SR. Inhibition, disinhibition, and the control of action in Tourette syndrome. Trends Cogn Sci 2015; 19: 655-65.

Jackson SR, Parkinson A, Kim SY, Schuermann M, Eickhoff SB. On the functional anatomy of the urge-for-action. Cogn Neurosci 2011; 2: 227-43.

Kessler RC, Adler L, Ames M, Demler O, Faraone S, Hiripi E, et al. The World Health Organization Adult ADHD Self-Report Scale (ASRS): a short screening scale for use in the general population. Psychol Med 2005; 35: 245-56.

Kwak C, Dat Vuong K, Jankovic J. Premonitory sensory phenomenon in Tourette's syndrome. Mov Disord 2003; 18: 1530-3.

Leckman JF, Riddle MA, Hardin MT, Ort SI, Swartz KL, Stevenson J, et al. The Yale Global Tic Severity Scale: initial testing of a clinicianrated scale of tic severity. J Am Acad Child Adolesc Psychiatr 1989; 28: 566-73.

Maia TV, Conceicao VA. The roles of phasic and tonic dopamine in tic learning and expression. Biol Psychiatr 2017; 82: 401-12.

Martino D, Ganos C, Pringsheim TM. Tourette syndrome and chronic Tic disorders: the clinical spectrum beyond Tics. Int Rev Neurobiol 2017; 134: 1461-90.

Matsuda N, Kono T, Nonaka M, Fujio M, Kano Y. Self-initiated coping with Tourette's syndrome: effect of tic suppression on QOL. Brain Dev 2016; 38: 233-41.

Mazzone L, Yu S, Blair C, Gunter BC, Wang Z, Marsh R, et al. An FMRI study of frontostriatal circuits during the inhibition of eye blinking in persons with Tourette syndrome. Am J Psychiatr 2010; 167: 341-9.

Nachev P, Kennard C, Husain M. Functional role of the supplementary and pre-supplementary motor areas. Nat Rev Neurosci 2008; 9 : 856-69.

Neuner I, Wegener P, Stoecker T, Kircher T, Schneider F, Shah NJ. Development and implementation of an MR-compatible whole body video system. Neurosci Lett 2007; 420: 122-7.

Neuner I, Werner CJ, Arrubla J, StÃ $\llbracket$ Cker T, Ehlen C, Wegener HP, et al. Imaging the where and when of Tic generation and resting state networks in adult Tourette patients. Front Hum Neurosci 2014; 8: 362 .

Parkinson J, Garfinkel S, Critchley H, Dienes Z, Seth AK. Don't make me angry, you wouldn't like me when I'm angry: volitional choices to act or inhibit are modulated by subliminal perception of emotional faces. Cogn Affect Behav Neurosci 2017; 17: 252-68.

Passingham RE, Bengtsson SL, Lau HC. Medial frontal cortex: from self-generated action to reflection on one's own performance. Trends Cogn Sci 2010; 14: 16-21.

Peterson BS, Skudlarski P, Anderson AW, Zhang H, Gatenby JC, Lacadie CM, et al. A functional magnetic resonance imaging study of tic suppression in Tourette syndrome. Arch Gen Psychiatr 1998; 55: 326-33.

Polyanska L, Critchley HD, Rae CL. Centrality of prefrontal and motor preparation cortices to Tourette syndrome revealed by metaanalysis of task-based neuroimaging studies. NeuroImage Clin 2017; 16: 257-67.

Rae CL, Ahmad A, Larsson DEO, Silva M, Praag CDGV, Garfinkel $\mathrm{SN}$, et al. Impact of cardiac interoception cues and confidence on 
voluntary decisions to make or withhold action in an intentional inhibition task. Sci Rep 2020; 10: 4184. doi: 10.1038/s41598020-60405-8.

Rae CL, Critchley HD, Seth AK. A bayesian account of the sensorymotor interactions underlying symptoms of Tourette syndrome. Front Psychiatr 2019; 10: 29.

Rae CL, Hughes LE, Anderson MC, Rowe JB. The prefrontal cortex achieves inhibitory control by facilitating subcortical motor pathway connectivity. J Neurosci 2015; 35: 786-94.

Rae CL, Hughes LE, Weaver C, Anderson MC, Rowe JB. Selection and stopping in voluntary action: a meta-analysis and combined fMRI study. Neuroimage 2014; 86: 381-91.

Rae CL, Polyanska L, Gould van Praag CD, Parkinson J, Bouyagoub $S$, Nagai $Y$, et al. Face perception enhances insula and motor network reactivity in Tourette syndrome. Brain 2018; 141: 3249-61.

Robertson MM. A personal 35 year perspective on Gilles de la Tourette syndrome: prevalence, phenomenology, comorbidities, and coexistent psychopathologies. Lancet Psychiatr 2015; 2: 68-87.

Robertson MM, Eapen V. Tourette's: syndrome, disorder or spectrum? Classificatory challenges and an appraisal of the DSM criteria. Asian J Psychiatr 2014; 11: 106-13.

Sebastian A, Forstmann BU, Matzke D. Towards a model-based cognitive neuroscience of stopping-a neuroimaging perspective. Neurosci Biobehav Rev 2018; 90: 130-6.

Thomalla G, Jonas M, Baumer T, Siebner HR, Biermann-Ruben K, Ganos C, et al. Costs of control: decreased motor cortex engagement during a Go/NoGo task in Tourette's syndrome. Brain 2014; 137: 122-36.
Tottenham N, Tanaka JW, Leon AC, McCarry T, Nurse M, Hare TA, et al. The NimStim set of facial expressions: judgments from untrained research participants. Psychiatr Res 2009; 168: 242-9.

van der Salm SMA, van der Meer JN, Cath DC, Groot PFC, van der Werf YD, Brouwers E, et al. Distinctive tics suppression network in Gilles de la Tourette syndrome distinguished from suppression of natural urges using multimodal imaging. Neuroimage Clin 2018; 20 : 783-92.

Woods DW, Piacentini J, Himle MB, Chang S. Premonitory Urge for Tics Scale (PUTS): initial psychometric results and examination of the premonitory urge phenomenon in youths with Tic disorders. J Dev Behav Pediatrics 2005; 26: 397-403.

Worbe Y, Marrakchi-Kacem L, Lecomte S, Valabregue R, Poupon F, Guevara P, et al. Altered structural connectivity of cortico-striatopallido-thalamic networks in Gilles de la Tourette syndrome. Brain 2015; 138: 472-82.

Zapparoli L, Porta M, Paulesu E. The anarchic brain in action: the contribution of task-based fMRI studies to the understanding of Gilles de la Tourette syndrome. Curr Opin Neurol 2015; 28: 604-11.

Zapparoli L, Seghezzi S, Paulesu E. The what, the when, and the whether of intentional action in the brain: a meta-analytical review. Front Hum Neurosci2017a; 11: 238.

Zapparoli L, Tettamanti M, Porta M, Zerbi A, Servello D, Banfi G, et al. A tug of war: antagonistic effective connectivity patterns over the motor cortex and the severity of motor symptoms in Gilles de la Tourette syndrome. Eur J Neurosci 2017b; 46: 2203-13.

Zhang J, Rittman T, Nombela C, Fois A, Coyle-Gilchrist I, Barker RA, et al. Different decision deficits impair response inhibition in progressive supranuclear palsy and Parkinson's disease. Brain 2016; 139: 161-73. 